\title{
Test of the consistency of various linearized semiclassical initial value time correlation functions in application to inelastic neutron scattering from liquid para-hydrogen
}

\author{
Jian Liu and William H. Miller \\ Department of Chemistry and K. S. Pitzer Center for Theoretical Chemistry \\ University of California, \\ and Chemical Science Division, Lawrence Berkeley National Laboratory
}

Berkeley, California 94720-1460 


\begin{abstract}
The linearized approximation to the semiclassical initial value representation (LSC-IVR) is
\end{abstract} used to calculate time correlation functions relevant to the incoherent dynamic structure factor for inelastic neutron scattering from liquid para-hydrogen at $14 \mathrm{~K}$. Various time correlations functions were used which, if evaluated exactly, would give identical results, but they do not because the LSC-IVR is approximate. Some of the correlation functions involve only linear operators, and others involve non-linear operators. The consistency of the results obtained with the various time correlation functions thus provides a useful test of the accuracy of the LSC-IVR approximation and its ability to treat correlation functions involving both linear and nonlinear operators in realistic anharmonic systems. The good agreement of the results obtained from different correlation functions, their excellent behavior in the spectral moment tests based on the exact moment constraints, and their semi-quantitative agreement with the inelastic neutron scattering experimental data all suggest that the LSC-IVR is indeed a good short-time approximation for quantum mechanical correlation functions. 


\section{Introduction}

Most quantities of interest in the dynamics of complex systems can be expressed in terms of thermal time correlation functions ${ }^{1}$. For example, dipole moment correlation functions are related to absorption spectra, flux correlation functions yield reaction rates, velocity correlation functions can be used to calculate diffusion constants, and vibrational energy relaxation rate constants can be expressed in terms of force correlation functions. These correlation functions ${ }^{1}$ are of the form

$$
C_{A B}(t)=\operatorname{Tr}\left(\hat{A}^{\beta} e^{i \hat{H} t / \hbar} \hat{B} e^{-i \hat{H} t / \hbar}\right),
$$

where $\hat{A}^{\beta}=\frac{1}{Z} e^{-\beta \hat{H}} \hat{A}$ for the standard version of the correlation function, or $\hat{A}_{\text {sym }}^{\beta}=\frac{1}{Z} e^{-\beta \hat{H} / 2} \hat{A} e^{-\beta \hat{H} / 2}$ for the symmetrized version ${ }^{2}$, or $\hat{A}_{\text {Kubo }}^{\beta}=\frac{1}{Z \beta} \int_{0}^{\beta} \mathrm{d} \lambda e^{-(\beta-\lambda) \hat{H}} \hat{A} e^{-\lambda \hat{H}}$ for the Kubo-transformed version ${ }^{3}$. These three versions are related to one another by the following identities between their Fourier transforms

$$
\frac{\beta \hbar \omega}{1-e^{-\beta \hbar \omega}} \tilde{C}_{A B}^{K u b o}(\omega)=\tilde{C}_{A B}(\omega)=e^{\beta \hbar \omega / 2} \tilde{C}_{A B}^{\text {sym }}(\omega),
$$

where $\tilde{C}_{A B}(\omega)=\frac{1}{2 \pi} \int_{-\infty}^{\infty} d t e^{-i \omega t} C_{A B}(t)$ etc. Here $\hat{H}$ is the (time-independent) Hamiltonian for the system, which for large molecular systems is usually expressed in terms of its Cartesian coordinates and momenta

$$
\widehat{H}=\frac{1}{2} \hat{\mathbf{p}}^{\mathrm{T}} \mathbf{M}^{-1} \hat{\mathbf{p}}+V(\hat{\mathbf{q}})=\hat{H}_{0}+V(\hat{\mathbf{q}})
$$

where $\mathbf{M}$ is the (diagonal) mass matrix and $\hat{\mathbf{p}}, \hat{\mathbf{q}}$ are the momentum and coordinate operators, respectively. Also, in Eq. (1.1) $Z=\operatorname{Tr}^{-\beta \hat{H}}\left(\beta=1 / k_{B} T\right)$ is the partition function, and $\hat{A}$ and $\hat{B}$ are operators relevant to the specific property of interest. 
For large molecular systems, classical molecular dynamics (MD) simulation methods are the only generally applicable approach, so for this reason we have been pursuing the use various initial value representations (IVRs) ${ }^{4-19}$ of semiclassical (SC) theory ${ }^{20,21}$ to add quantum effects to classical MD simulations of time correlation functions. The SC-IVR provides a way for generating the quantum time evolution operator (propagator) $e^{-i \hat{H} t / \hbar}$ by computing an ensemble of classical trajectories, much as is done in standard classical molecular dynamics simulations. Such approaches actually contains all quantum effects at least qualitatively, and in molecular systems the description is usually quite quantitative $\mathrm{e}^{4-8,15,20-27}$.

The simplest (and most approximate) version of the SC-IVR is its 'linearized' approximation (LSC-IVR) $^{9,26,28-35}$, which leads to the classical Wigner model ${ }^{36-39}$ for time correlation functions; see Section IIB for a summary of the LSC-IVR. The classical Wigner model is an old idea, but it is important to realize that it is contained within the SC-IVR approach, as a well-defined approximation to it ${ }^{28,29}$. There are other ways to derive the classical Wigner model (or one may simply postulate it) $)^{9,35,40,41}$, and we also note that the 'forward-backward semiclassical dynamics' (FBSD) approximation of Makri et al..$^{32,42-56}$ is very similar to it. The LSC-IVR/classical Wigner model cannot describe true quantum coherence effects in time correlation functions-more accurate SC-IVR approaches, such as the Fourier transform forward-backward IVR (FB-IVR) approach ${ }^{22,57}$ (or the still more accurate generalized FB-IVR ${ }^{58}$ ) of Miller et al., are needed for this-but it does describe some aspects of the quantum dynamics very well ${ }^{26,30-32,34,59-62}$. E.g., the LSC-IVR has been shown to 
describe reactive flux auto-correlation functions (which determine chemical reaction rates) quite well, including strong tunneling regimes ${ }^{31}$, and velocity auto-correlation functions ${ }^{26,32,60}$ and force auto-correlation functions ${ }^{26,34,61,62}$ in systems with enough degrees of freedom for quantum re-phasing to be unimportant.

Similar to the LSC-IVR are two other ways to approximate the quantum dynamic correlation function such that the result both approaches its classical limit at high temperature and achieves the exact quantum result as $t \rightarrow 0$ for arbitrary potentials. One such approach is the centroid molecular dynamics (CMD) method of Voth and co-workers ${ }^{63-75}$, and another is the ring polymer molecular dynamics (RPMD) model recently proposed by Manolopoulos and co-workers ${ }^{76-81}$. In these approaches the real time dynamics is related to a modified classical dynamics of the path integral beads of the quantum Boltzmann operator or the centroid of them. These two models are also unable to capture true quantum coherence effects. For the case of harmonic systems, both of these models give the exact quantum result if at least one of the operators $\hat{A}$ and $\hat{B}$ is a linear function of position or momentum operators; however, they do not give the correct result if both operators are non-linear ${ }^{71,78,82,83}$; the LSC-IVR, on the other hand, gives the exact quantum correlation function for all time $t$ and for arbitrary operators $\hat{A}$ and $\hat{B}$ for a harmonic potential ${ }^{9}$. Fig. 4 of a recent study ${ }^{26}$ shows that for the realistic anharmonic system liquid para- $\mathrm{H}_{2}$ at the state point (25K under nearly zero external pressure), the LSC-IVR is a more faithful approximation to quantum mechanical real time correlation functions at short time (on the order of thermal time $\hbar \beta$ ) than the CMD and RPMD 
models even for linear operators (such as $\hat{\mathbf{p}}$ or $\hat{\mathbf{x}}$ ). How generally true this conclusion is must of course await future investigations on other realistic systems. However, both the CMD and the RPMD models have the desirable feature that the quantum mechanical equilibrium distribution is correctly conserved-i.e., for the case $\hat{A}=1$ the correlation function (i.e. the canonical ensemble average of operator $\hat{B}$ ) is time-independent-while this is not the case for the LSC-IVR (though Liu et $a l .{ }^{26,54}$ have demonstrated that this is in fact not a problem in practical calculations so long as the correlation time scale is not too long).

We also note here that the maximum entropy analytic continuation (MEAC) approach developed mainly by Berne and coworkers ${ }^{84-89}$ and quantum mode-coupling theory (QMCT) approach of Rabani and Reichman ${ }^{90-97}$ are also very useful methods to capture accurate short-time behavior of the real time correlation function. Since only the imaginary time information is needed as the input, calculations of these two methods are usually light and are feasible for cases where dynamics are very slow (i.e., glassy liquids), which is the strength of these two methods. However, both methods have their shortcomings as well. I.e, neither of them is exact in the classical limit (although the QMCT reaches the classical mode-coupling theory that is accurate in many cases in the classical limit), the MEAC is not so good when the spectrum of the correlation function has multiple maxima ${ }^{87}$ and when the system has a separation of time scales, and the mode-coupling theory is not easy to apply to polyatomic liquids ${ }^{98,99}$. Since this paper mainly discusses on the approximated quantum dynamical methods involving trajectories, we focus on the comparison among the LSC-IVR, CMD and RPMD. 
The purpose of this paper is to present an additional challenging application and test of the LSC-IVR approximation to quantum mechanical time correlation functions, namely the incoherent dynamic structure factor for inelastic neutron scattering from liquid para-hydrogen ${ }^{100,101}$, with special emphasis on how consistent the results are when obtaining this quantity from various time correlation functions. I.e., in most cases the physical quantity of interest can be expressed in terms of different time correlation functions, which would all give the same result if the calculations could be carried out exactly: e.g., diffusion coefficients can be obtained from position-position or velocity-velocity correlation functions, rate constants can be obtained from flux-flux or side-side correlation functions, etc. When the calculations are carried out approximately, though, the results for the physical quantity given by using different correlation functions will generally be different, and the degree to which they do agree with each other thus offers some measure of how accurate one believes the approximate treatment to be. In the present case, the incoherent dynamic structure factor for inelastic neutron scattering can be obtained from the self-part of the intermediate scattering function (involving nonlinear operators), or from the velocity correlation function (involving linear operators) $^{102}$; see Section IIA for more details. This thus provides an ideal test case to study the behavior of the LSC-IVR method and its comparison to the $\mathrm{CMD}^{72}$ and the RPMD ${ }^{78}$ models. Section II first summarizes the theory of inelastic neutron scattering and shows how the self-part of the intermediate scattering function and the velocity correlation function are related with each other, and then describes the LSC-IVR formulation of these time correlation functions using the thermal 
Gaussian approximation (TGA) ${ }^{26,32}$. Section III presents the LSC-IVR simulation results for the incoherent dynamic structure factor of liquid para-hydrogen at $T=14 \mathrm{~K}$ (under nearly zero external pressure) using different correlation functions, along with the spectral moment test and the comparison to other methods and the recent inelastic neutron scattering experiment data ${ }^{101}$. Conclusions are given in Section IV.

\section{Theory and Methodology}

\section{A. Inelastic Neutron Scattering}

Inelastic neutron scattering is a well established technique for obtaining information on dynamic structure of liquids and vibrational spectroscopy ${ }^{103-105}$. Within the first Born approximation, the experimentally observed differential scattering cross section was shown by Von Hove ${ }^{106}$ to be proportional to the coherent dynamic structure factor which reflects the collective behavior of liquids

$$
S_{c o h}(\kappa, \omega)=\frac{1}{2 \pi} \int_{-\infty}^{\infty} d t e^{-i \omega t} F(\mathbf{\kappa}, t)
$$

Here the intermediate scattering function $F(\boldsymbol{\kappa}, t)$ is given by

$$
F(\boldsymbol{\kappa}, t)=\frac{1}{N} \sum_{i, j=1}^{N}\left\langle e^{-i \kappa \cdot \hat{x}_{i}} e^{i \kappa \cdot \hat{\mathbf{x}}_{j}(t)}\right\rangle
$$

where $N$ is the number of particles of the system, $\hat{\mathbf{x}}_{i}$ is the position operator of the $i$-th particle, $e^{i \boldsymbol{\kappa} \cdot \hat{\mathbf{x}}_{j}(t)}=e^{i \hat{H} t / \hbar} e^{i \boldsymbol{\kappa} \cdot \hat{\mathbf{x}}_{j}} e^{-i \hat{H} t / \hbar}$ is the Heisenberg operator of $e^{i \boldsymbol{\kappa} \cdot \hat{\mathbf{x}}_{j}}$, and the momentum and energy transfers from the scattered neutron to the liquid are respectively

$$
\begin{gathered}
\hbar \mathbf{\kappa}=\hbar \boldsymbol{\kappa}_{i}-\hbar \boldsymbol{\kappa}_{f} \\
\hbar \omega=\frac{\hbar^{2}\left(\boldsymbol{\kappa}_{i}^{2}-\boldsymbol{\kappa}_{f}^{2}\right)}{2 m_{n}}
\end{gathered}
$$


with $\boldsymbol{\kappa}_{i}$ and $\boldsymbol{\kappa}_{f}$ are the initial and the final wave vectors of the neutron, and $m_{n}$ is the mass of the neutron.

For some liquids, such as liquid hydrogen and deuterium, in which the particles have nuclear spin effects or nuclear internal variables, the incoherent dynamic structure factor which reflects the single-particle motion is pronounced ${ }^{104}$,

$$
S_{i n c}(\kappa, \omega)=\frac{1}{2 \pi} \int_{-\infty}^{\infty} d t e^{-i \omega t} F_{s}(\kappa, t),
$$

where the self-part of the intermediate scattering function is

$$
F_{S}(\boldsymbol{\kappa}, t)=\frac{1}{N} \sum_{i=1}^{N}\left\langle e^{-i \kappa \cdot \hat{\mathbf{x}}_{i}(0)} e^{i \boldsymbol{\kappa} \cdot \hat{\mathbf{x}}_{i}(t)}\right\rangle
$$

For isotropic systems, both the dynamic structure factors and the scattering functions only depend on $\kappa=|\boldsymbol{\kappa}|$, i.e., they are independent of the direction of $\boldsymbol{\kappa}$.

Using a cumulant expansion, it can be shown that the self-part of the intermediate scattering function has the following equivalent form ${ }^{102}$

$$
F_{s}(\boldsymbol{\kappa}, t)=\exp \left[i t \frac{\hbar \kappa^{2}}{2 m}+\sum_{n=1}^{\infty}\left(-\kappa^{2}\right)^{n} \gamma_{n}(t)\right]
$$

where $m$ is the mass of the particle in the pure liquid, and $\gamma_{n}(t)$ is related to $2 n$-point velocity correlation functions, i.e.,

$$
\begin{gathered}
\gamma_{1}(t)=\int_{0}^{t} d t_{1} \int_{0}^{t_{1}} d t_{2}\left\langle v_{\kappa}\left(t_{2}\right) v_{\kappa}\left(t_{1}\right)\right\rangle, \\
\gamma_{2}(t)=\int_{0}^{t} d t_{1} \int_{0}^{t_{1}} d t_{2} \cdots \int_{0}^{t_{3}} d t_{4}\left\langle v_{\kappa}\left(t_{4}\right) \cdots v_{\kappa}\left(t_{1}\right)\right\rangle-\frac{1}{2}\left[\gamma_{1}(t)\right]^{2},
\end{gathered}
$$

and so on, where $v_{\kappa}$ is the velocity component along the direction of $\boldsymbol{\kappa}$. For small values of $\kappa$, the first order truncation in Eq. (2.7) gives a Gaussian approximation, which for isotropic liquids is 


$$
F_{s}(\kappa, t)=\exp \left[\kappa^{2}\left(i t \frac{\hbar}{2 m}-\frac{1}{3} \int_{0}^{t}\left(t-t^{\prime}\right) C_{\mathbf{v v}}\left(t^{\prime}\right) d t^{\prime}\right)\right],
$$

where $C_{\mathrm{vv}}\left(t^{\prime}\right)$ is the standard velocity auto-correlation function given by Eq. (1.1). For the case that the velocity distribution of the system is Gaussian, $\gamma_{2}(t)$ and higher order terms vanish, so that the Gaussian approximation of Eq (2.10) becomes exact. Furthermore, it can be shown that the velocity distribution is Gaussian (i.e., Maxwellian) even if quantum corrections through order $\hbar^{2}$ are taken into account ${ }^{107}$, so that higher order corrections to the Gaussian approximation of Eq. (2.10) are expected to be extremely small even for large $\kappa$, except for very low temperatures ${ }^{102}$. Eq. (2.10) is in fact a very good approximation, i.e., non-Maxwellian effects are indeed negligible, for the system under study in this paper-liquid para-hydrogen at $14 \mathrm{~K}$--as implied in the literature $\mathrm{r}^{72,78,100}$ and also discussed in Section III and Appendix C. The incoherent dynamic structure factor can thus be computed either directly through the self-part of the intermediate scattering function, Eq. (2.6), or indirectly through the standard velocity function, Eq. (2.10), thus providing a test of the consistency of the LSC-IVR for these different correlation functions (involving both linear and nonlinear operators).

\section{B. LSC-IVR Correlation Functions Using the TGA}

The SC-IVR approximates the forward (backward) time evolution operator $e^{-i \hat{H} t / \hbar}\left(e^{i \hat{H} t / \hbar}\right)$ by a phase space average over the initial conditions of forward (backward) classical trajectories ${ }^{5,7,8,20}$. By making the (drastic but reasonable) approximation that the dominant contribution to the phase space averages comes from forward and backward trajectories that are close to one another and then 
linearizing the forward and backward actions of such trajectories, Miller and coworkers ${ }^{28-30}$ obtained the linearized SC-IVR (LSC-IVR), or classical Wigner model for the correlation function

$$
C_{A B}^{L S C-I V R}(t)=(2 \pi \hbar)^{-3 N} \int d \mathbf{x}_{0} \int d \mathbf{p}_{0} A_{w}^{\beta}\left(\mathbf{x}_{0}, \mathbf{p}_{0}\right) B_{w}\left(\mathbf{x}_{t}, \mathbf{p}_{t}\right)
$$

where $A_{w}^{\beta}$ and $B_{w}$ are the Wigner functions ${ }^{36}$ corresponding to these operators,

$$
O_{w}(\mathbf{x}, \mathbf{p})=\int d \boldsymbol{\Delta} \mathbf{x}\langle\mathbf{x}-\Delta \mathbf{x} / 2|\hat{O}| \mathbf{x}+\Delta \mathbf{x} / 2\rangle e^{i \mathbf{p}^{T} \Delta \mathbf{x} / \hbar}
$$

for any operator $\hat{O}$. Here $\left(\mathbf{x}_{0}, \mathbf{p}_{0}\right)$ is the set of initial conditions (i.e., coordinates and momenta) for a classical trajectory, $\left(\mathbf{x}_{t}\left(\mathbf{x}_{0}, \mathbf{p}_{0}\right), \mathbf{p}_{t}\left(\mathbf{x}_{0}, \mathbf{p}_{0}\right)\right)$ being the phase point at time $t$ along that trajectory. More recently, Liu and Miller $^{9}$ have shown that the exact quantum time correlation function can be expressed in the same form as Eq. (2.11), with an associated dynamics in the single phase space, and it was furthermore demonstrated that the LSC-IVR is its classical limit $(\hbar \rightarrow 0)$, high temperature limit $(\beta \rightarrow 0)$, and harmonic limit. The LSC-IVR can be applied not only to correlation functions at equilibrium but also to non-equilibrium correlation functions. These merits of the LSC-IVR make it a versatile tool to study quantum-mechanical effects in chemical dynamics of large molecular systems.

Here we use the thermal Gaussian approximation ${ }^{108-110,111 \text {; Shao, } 2006 \# 1741}$ (TGA) of Frantsuzov and Mandelshtam to construct the Boltzmann operator as necessary to obtain the Wigner function of operator $\hat{A}_{\beta}{ }^{26,32}$. In the TGA, the Boltzmann matrix element is approximated by a Gaussian form:

$$
\left\langle\mathbf{x}\left|e^{-\tau \hat{H}}\right| \mathbf{q}_{\mathbf{0}}\right\rangle=\left(\frac{1}{2 \pi}\right)^{3 N / 2} \frac{1}{|\operatorname{det}(\mathbf{G}(\tau))|^{1 / 2}} \exp \left(-\frac{1}{2}(\mathbf{x}-\mathbf{q}(\tau))^{T} \mathbf{G}^{-1}(\tau)(\mathbf{x}-\mathbf{q}(\tau))+\gamma(\tau)\right),
$$


where $\mathbf{G}(\tau)$ is an imaginary-time dependent real symmetric and positive-definite matrix, $\mathbf{q}(\tau)$ the center of the Gaussian, and $\gamma(\tau)$ a real scalar function. The parameters are governed by the equations of motion in imaginary time which were given explicitly in our previous paper ${ }^{32}$ and in other references $^{108,109,112}$. The matrix $\mathbf{G}(\tau)$ is a full $3 N \times 3 N$ matrix, where $N$ is number of particles of the system. The TGA for the Boltzmann operator makes it possible to perform the Fourier transform necessary to construct the Wigner function of operator $\hat{A}^{\beta}$ analytically; specifically, $A_{w}^{\beta}\left(\mathbf{x}_{0}, \mathbf{p}_{0}\right)$ in Eq. (2.11) is given as follows ${ }^{32}$

$$
\begin{aligned}
A_{w}^{\beta}\left(\mathbf{x}_{0}, \mathbf{p}_{0}\right)= & \frac{1}{Z} \int d \mathbf{q}_{0} \frac{1}{(4 \pi)^{3 N / 2}} \frac{\exp \left(2 \gamma\left(\frac{\beta}{2}\right)\right)}{\left|\operatorname{det} \mathbf{G}\left(\frac{\beta}{2}\right)\right|^{1 / 2}} \\
& \cdot \frac{1}{\pi^{3 N / 2}\left|\operatorname{det} \mathbf{G}\left(\frac{\beta}{2}\right)\right|^{1 / 2}} \exp \left(-\left(\mathbf{x}_{0}-\mathbf{q}\left(\frac{\beta}{2}\right)\right)^{T} \mathbf{G}^{-1}\left(\frac{\beta}{2}\right)\left(\mathbf{x}_{0}-\mathbf{q}\left(\frac{\beta}{2}\right)\right)\right), \\
& \cdot \frac{\left|\operatorname{det} \mathbf{G}\left(\frac{\beta}{2}\right)\right|^{1 / 2}}{\left(\pi \hbar^{2}\right)^{3 N / 2}} \exp \left(-\mathbf{p}_{0}^{T} \mathbf{G}\left(\frac{\beta}{2}\right) \mathbf{p}_{0} / \hbar^{2}\right) \\
& \cdot f_{A^{\beta}}^{T G A}\left(\mathbf{x}_{0}, \mathbf{p}_{0}, \mathbf{q}\left(\frac{\beta}{2}\right)\right)
\end{aligned}
$$

where for the Kubo-transformed momentum correlation function

$$
f_{A^{\beta}, \text { Kubo }}^{\text {TGA-LSC-IVR }}\left(\mathbf{x}_{0}, \mathbf{p}_{0}, \mathbf{q}\left(\frac{\beta}{2}\right)\right)=\frac{2}{\hbar^{2} \beta} \mathbf{M G}\left(\frac{\beta}{2}\right) \mathbf{p}_{0}
$$

for the momentum operator $\hat{A}=\hat{\mathbf{p}}$ with $\hat{A}_{\text {Kubo }}^{\beta}=\frac{1}{Z \beta} \int_{0}^{\beta} d \lambda e^{-(\beta-\lambda) \hat{H}} \hat{\mathbf{p}} e^{-\lambda \hat{H}}{ }^{32}$; for the standard momentum correlation function

$$
f_{A A}^{T G A-L S C-I V R}\left(\mathbf{x}_{0}, \mathbf{p}_{0}, \mathbf{q}\left(\frac{\beta}{2}\right)\right)=\mathbf{p}_{0}-i \hbar \mathbf{G}^{-1}\left(\frac{\beta}{2}\right)\left(\mathbf{x}_{0}-\mathbf{q}\left(\frac{\beta}{2}\right)\right)
$$

for the momentum operator $\hat{A}=\hat{\mathbf{p}}$ with $\hat{A}^{\beta}=\frac{1}{Z} e^{-\beta \hat{H}} \hat{\mathbf{p}}^{32}$; for the self-part of the intermediate scattering function 


$$
\begin{aligned}
& f_{A A}^{\text {TGA-LSC-IVR }}\left(\mathbf{x}_{0}, \mathbf{p}_{0}, \mathbf{q}\left(\frac{\beta}{2}\right)\right)= \\
& \frac{1}{N} \sum_{i=1}^{N} e^{-i \boldsymbol{\kappa} \cdot \mathbf{x}_{i}(0)} \exp \left[\mathbf{p}_{0}^{T} \cdot \mathbf{G}_{\mathbf{x}_{i}}\left(\frac{\beta}{2}\right) \cdot \mathbf{\kappa} / \hbar-\frac{1}{4} \boldsymbol{\kappa}^{T} \cdot \mathbf{G}_{\mathbf{x}_{i} \mathbf{x}_{i}}\left(\frac{\beta}{2}\right) \cdot \mathbf{\kappa}\right]
\end{aligned}
$$

for the operator $\hat{A}=\frac{1}{N} \sum_{i=1}^{N} e^{-i \boldsymbol{\kappa} \cdot \hat{\mathbf{x}}_{i}}$ with $\hat{A}^{\beta}=\frac{1}{Z} e^{-\beta \hat{H}}\left(\frac{1}{N} \sum_{i=1}^{N} e^{-i \boldsymbol{\kappa} \cdot \hat{\mathbf{x}}_{i}}\right)$. Here $\mathbf{G}_{\mathbf{x}_{i}}\left(\frac{\beta}{2}\right)$ denotes the three columns (related with $\mathbf{x}_{j}$ ) of the matrix $\mathbf{G}\left(\frac{\beta}{2}\right)$, and $\mathbf{G}_{\mathbf{x}_{i} \mathbf{x}_{i}}\left(\frac{\beta}{2}\right)$ the $3 \times 3$ block matrix of which the rows and columns representing $\mathbf{x}_{j}$. The derivation of Eq. (2.17) is shown in Appendix A.

Calculation of $B_{w}$ in Eq. (2.11) is usually an easy task; in fact, $\hat{B}$ is often a function only of coordinates or only of momenta, in which case its Wigner function is simply the classical function itself. Monte Carlo (MC) evaluation of Eq. (2.11) together with Eq. (2.14) is now straightforward, and we refer readers to Section IV of our recent paper $^{32}$ for more details. We note here that the TGA/LSC-IVR is exact in the classical limit and in the harmonic limit as pointed out in our previous work $^{32}$.

For our simulations (the results of which are presented and discussed in Section III) we have used the following three approaches to calculate the incoherent dynamic structure factor $S_{\text {inc }}(\kappa, \omega)$ :

1. Direct implementation of the TGA/LSC-IVR in Eqs. (2.11), (2.14), and (2.17) to calculate the self-part of the intermediate scattering function $F_{s}(\kappa, t)$, with $S_{\text {inc }}(\boldsymbol{\kappa}, \omega)$ then given by Eq. (2.5). We refer to this as "inelastic-std".

2. Use of Eqs. (2.11), (2.14) and (2.16) to obtain the standard velocity correlation function $C_{\mathbf{v} \cdot \mathbf{v}}(t)$ and then calculation of $S_{\text {inc }}(\boldsymbol{\kappa}, \omega)$ via Eqs. (2.10) and (2.5). We denote this “vv-std". 
3. Calculation of the Kubo-transformed velocity correlation function $C_{\mathbf{v} \cdot \mathbf{v}}^{\text {Kubo }}(t)$ by Eqs. (2.11), (2.14), and (2.15), then use of the relation between the spectra, Eq. (1.2), to obtain the standard correlation function, i.e.,

$$
C_{\mathbf{v} \cdot \mathbf{v}}(t)=\frac{1}{2 \pi} \int_{-\infty}^{\infty} d \omega e^{i \omega t} \frac{\beta \hbar \omega}{1-e^{-\beta \hbar \omega}} \int_{-\infty}^{\infty} d t^{\prime} e^{-i \omega t^{\prime}} C_{\mathbf{v} \cdot \mathbf{v}}^{K u b o}\left(t^{\prime}\right)
$$

with $S_{\text {inc }}(\kappa, \omega)$ then given by Eqs. (2.18), (2.10), and (2.5). We denote this approach “vv-kubo".

Though all three approaches above would give the same result for $S_{\text {inc }}(\boldsymbol{\kappa}, \omega)$ if the quantum mechanical correlation functions were calculated exactly, the results will actually be somewhat different because the LSC-IVR is being used to calculate the correlation functions. Comparing the results obtained for $S_{i n c}(\boldsymbol{\kappa}, \omega)$ by these various approaches thus provides a test of the consistency (and presumably the accuracy) of the LSC-IVR approximation for these correlation functions, which involve both linear and nonlinear operators.

\section{Spectral Moment Tests}

At present, exact quantum results of $S_{i n c}(\kappa, \omega)$ for this system are not available, so there is no way to be absolutely certain how well the LSC-IVR approximation performs in our present calculations. However low order spectral moments of $S_{\text {inc }}(\boldsymbol{\kappa}, \omega)$ can be calculated essentially exactly, by Feynman (imaginary time) path integrals methods, and this provides some rigorous comparisons by which to judge the accuracy of these methods. Define the recoil frequency as $\omega_{R}=\hbar \kappa^{2} / 2 m$. The now standard procedure ${ }^{102}$ is to express the spectral moments as 


$$
\mu_{n}(\mathbf{\kappa})=\int_{-\infty}^{\infty}\left(\omega-\omega_{R}\right)^{n} S_{i n c}(\mathbf{\kappa}, \omega) d \omega \equiv i^{-n} \frac{d^{n}}{d t^{n}}\left[e^{-i \omega_{R} t} F_{s}(\mathbf{\kappa}, t)\right]_{t=0}
$$

e.g., the three lowest moments for the isotropic system are ${ }^{102,113}$

$$
\begin{aligned}
& \mu_{0}(\kappa)=1, \\
& \mu_{1}(\kappa)=0,
\end{aligned}
$$

and

$$
\mu_{2}(\kappa)=\kappa^{2}\left\langle v_{\kappa}^{2}\right\rangle=\frac{\kappa^{2}}{3} C_{\mathbf{v} \cdot \mathbf{v}}(0) \equiv \frac{2 \kappa^{2}}{3 m}\left\langle\frac{\hat{\mathbf{p}}^{2}}{2 m N}\right\rangle
$$

It is straightforward to verify that, when the Gaussian approximation, Eq. (2.10), is combined with exact velocity correlation functions, all moments in Eq. (2.19) are exact if the velocity distribution of the system is Gaussian; the four lowest moments are exact even for more general velocity distributions. Eqs. (2.7) and (2.19) indicate that $\mu_{0}(\kappa)$ and $\mu_{1}(\kappa)$ remain exact when the Gaussian approximation, Eq. (2.10), is combined with any approximate velocity correlation function, but $\mu_{2}(\kappa)$ and higher order moments generally do not. Moreover, in Appendix B it is shown that $\mu_{0}(\kappa)$ and $\mu_{1}(\kappa)$ are also exact for the TGA/LSC-IVR formulation of the self-part of the intermediate scattering function $F_{s}(\kappa, t)$, Eq. (2.6). In summary, all three methods in Section IIB (the "inelastic-std", "vv-kubo" and "vv-std" methods based on the TGA/LSC-IVR) give the exact values for the two lowest moments $\mu_{0}(\kappa)$ and $\mu_{1}(\kappa)$ (as shown in Table 1)

Craig and Manolopoulos ${ }^{78}$ proposed another test to check the accuracy of $S_{\text {inc }}(\boldsymbol{\kappa}, \omega)$ by calculating another set of spectral moments of the incoherent relaxation spectrum $\tilde{S}_{\text {inc }}(\boldsymbol{\kappa}, \omega)$,

$$
\underline{\mu}_{n}(\mathbf{\kappa})=\int_{-\infty}^{\infty} \omega^{n} \tilde{S}_{i n c}(\boldsymbol{\kappa}, \omega) d \omega \equiv i^{-n} \frac{d^{n}}{d t^{n}}\left[\tilde{F}_{s}(\boldsymbol{\kappa}, t)\right]_{t=0} .
$$


Here the incoherent relaxation function $\tilde{F}_{s}(\kappa, t)$ is

$$
\tilde{F}_{s}(\boldsymbol{\kappa}, t)=\frac{1}{\beta N} \int_{0}^{\beta} d \lambda \sum_{i=1}^{N}\left\langle e^{-i \kappa \cdot \hat{r}_{i}(-i \hbar \lambda)} e^{i \kappa \cdot \hat{r}_{i}(t)}\right\rangle
$$

and $\tilde{S}_{\text {inc }}(\boldsymbol{\kappa}, \omega)$ is the Fourior transform of $\tilde{F}_{s}(\boldsymbol{\kappa}, t)$. Since we don't directly calculate $\tilde{F}_{s}(\boldsymbol{\kappa}, t)$ (and then its spectrum $\tilde{S}_{\text {inc }}(\boldsymbol{\kappa}, \omega)$ ) using the TGA/LSC-IVR, we implement the relation in Eq. (1.2) into Eq. (2.23), i.e.,

$$
\underline{\mu}_{n}(\boldsymbol{\kappa})=\int_{-\infty}^{\infty} \omega^{n} \frac{\left(1-e^{-\beta \hbar \omega}\right)}{\beta \hbar \omega} S_{i n c}(\boldsymbol{\kappa}, \omega) d \omega .
$$

It can be seen that each of the moment $\underline{\mu}_{n}(\mathbf{\kappa})$ in Eq. (2.25) involves the collection of the moments $\mu_{n}(\kappa)$ in Eq. (2.19). Based on the detailed balance for $S_{\text {inc }}(\kappa, \omega)$,

$$
e^{-\beta \hbar \omega} C_{A B}(\omega)=C_{A B}(-\omega) \text {, }
$$

one can show that all odd moments in Eq. (2.25) vanish. From Eq. (2.23), it can be shown ${ }^{3}$ that the first two even moments are

$$
\underline{\mu}_{0}(\kappa)=\tilde{F}_{S}(\kappa, 0) \equiv \chi_{S}(\kappa)
$$

and

$$
\underline{\mu}_{2}(\kappa)=\frac{\kappa^{2}}{\beta m}
$$

Accurate values ${ }^{78}$ of the susceptibility function $\chi_{S}(\kappa)$ can be obtained by imaginary time path integral techniques. Generally, all the three methods in Section IIB ("inelastic-std", "vv-kubo" and "vv-std" based on the TGA/LSC-IVR approach) only give approximate results for $\underline{\mu}_{0}(\kappa)$ and $\underline{\mu}_{2}(\kappa)$ (see Table 1). Comparison of these results with the exact ones can thus be used to check the accuracy of $S_{\text {inc }}(\boldsymbol{\kappa}, \omega)$ given by the three methods proposed in Section IIB. Table 1 (for the 
LSC-IVR) in this paper can be directly compared with Table 1 (for the RPMD) in Ref. ${ }^{78,114}$, though such a table the CMD is not available in Ref. ${ }^{72}$.

\section{Results and Discussions}

\section{A. Simulation details}

The system under study is liquid para-hydrogen at the state point $T=14 \mathrm{~K}, v=23.5 \mathrm{~nm}^{-3}$ under nearly zero external pressure ${ }^{115}$, for which the Kubo-transformed velocity correlation function has been calculated in our previous paper ${ }^{26}$. The computational details are quite similar and are briefly described as follows.

Liquid para-hydrogen is well described by the Silvera-Goldman (SG) model ${ }^{116}$, an isotropic pair potential in which the para-hydrogen molecule is treated as a sphere particle. Thus both $S_{\text {inc }}(\boldsymbol{\kappa}, \omega)$ and $F_{s}(\boldsymbol{\kappa}, t)$ depend only on $\kappa=|\boldsymbol{\kappa}|$. To accelerate the imaginary time propagation in the TGA, we fit the SG potential to a linear combination of Gaussians ${ }^{26}$. In the simulation, we used periodic boundary conditions with 108 molecules per cell with the minimum image convention at various values of the momentum transfer parameter $\kappa=|\mathbf{\kappa}|$ that satisfy the Laue relation ${ }^{117,118}$

$$
\kappa=2 n \pi / L,
$$

where $L$ is the length of the unit cell and $n$ is integer. As in our previous applications ${ }^{26,32}$, the standard Metropolis algorithm was implemented and the acceptance ratio of new initial Gaussians (for the Boltzmann matrix element, Eq. (2.13)) was about 40\%. The initial inverse temperature of starting Gaussians was $0.0001 \beta$. About $5 \times 10^{4}$ imaginary trajectories were used for initial equilibrations, and then during the simulation of the correlation function the total number of 
imaginary trajectories was $8.6 \times 10^{5}$, with an imaginary time step of 22 . With initial conditions generated by each imaginary time trajectory, 10 real time trajectories were propagated with the usual velocity Verlet algorithm, with a time step of $1.2 \mathrm{fs}$.

During the TGA/LSC-IVR simulation, $F_{s}(\boldsymbol{\kappa}, t), C_{\mathbf{v} \cdot \mathbf{v}}(t)$ and $C_{\mathbf{v} \cdot \mathbf{v}}^{\text {Kubo }}(t)$ were calculated simultaneously by collecting their estimators $f_{A^{\beta}}^{T G A}\left(\mathbf{x}_{0}, \mathbf{p}_{0}, \mathbf{q}\left(\frac{\beta}{2}\right)\right) \cdot B_{w}\left(\mathbf{x}_{t}, \mathbf{p}_{t}\right)$ along trajectories. For convenience, the incoherent dynamic structure factor $S_{i n c}(\kappa, \omega)$ was calculated from the real part of $F_{s}(\kappa, t)$, i.e.,

$$
S_{\text {inc }}(\boldsymbol{\kappa}, \omega)=\frac{1}{\pi\left(1+e^{-\beta \hbar \omega}\right)} \int_{-\infty}^{\infty} d t e^{-i \omega t} \operatorname{Re}\left[F_{s}(\boldsymbol{\kappa}, t)\right]
$$

It is straightforward to derive Eq. (3.2) based on the detail balance, Eq. (2.26).

\section{B. Incoherent Dynamic Structure Factors}

Fig. 1 shows the self-part of the intermediate scattering function $F_{s}(\kappa, t)$ at four different values of the momentum transfer parameter, i.e., $\kappa=0.378 \AA^{-1}(n=1), \quad 1.512 \AA^{-1}(n=4)$, $2.646 \AA^{-1}(n=7)$ and $4.536 \AA^{-1}(n=12)$. One sees that the time scale for the decay of $F_{s}(\kappa, t)$ decreases as the momentum transfer parameter $\kappa$ increases. By way of comparison, the typical time scale of the Kubo-transformed velocity correlation function is $\leq 1 \mathrm{ps}$ as shown in Fig. 3b of Ref. $^{26}$. As pointed out in previous sections, the LSC-IVR approximation to quantum mechanical correlation functions is expected to be best at short times so that one would thus expect it to be better for larger momentum transfer. On the other hand, the larger the momentum transfer parameter $\kappa$, the more nonlinear are the operators in the correlation function $F_{s}(\kappa, t)$. Although it has already 
been shown that the LSC-IVR deals well ${ }^{26}$ with linear operators (i.e., the velocity correlation function) in this highly anharmonic system, there is still the question of how well it treats these nonlinear operators.

To check these two points, incoherent dynamic structure factors $S_{\text {inc }}(\boldsymbol{\kappa}, \omega)$ are calculated by the three methods proposed in Section IIB and are plotted as a function of the energy transfer parameter $\omega$ at various values of the momentum transfer parameter $\kappa$ in Fig. 2. (Appendix C discusses in more detail why Eq. (2.10) is expected to be a good approximation for all $\kappa$ for this system, and why this leads to the incoherent dynamic structure factor $S_{i n c}(\kappa, \omega)$ being Gaussian at very large $\kappa$ and Lorentzian at very small $\kappa$, as observed in Fig. 2.)

The most important conclusion from Fig. 2 is that the results of the three methods based on the LSC-IVR approximation are in very good agreement with one another, provided $\kappa$ is not very small. It is very encouraging that the results agree well with each other even for quite large values of $\kappa$, for which the relevant operators are highly non-linear. This demonstrates that the LSC-IVR provides a consistent approximation to the quantum mechanical correlation functions for both linear and the nonlinear operators when the time scale of the correlation function is not too long. However, for very small $\kappa$, corresponding to long time, Fig. 2 does show some deviations among the three methods proposed in Section IIB based on the TGA/LSC-IVR, although the peaks are located at nearly the same frequency. In this regime, the deviations among three methods imply that some 
inconsistency may exist in the LSC-IVR formulation of correlation functions for different operators at very long times.

Fig. 2 can be directly compared with Fig. 7 in Ref. ${ }^{72}$ by Hone and Voth, and Fig. 1 in Ref. ${ }^{78}$ by Craig and Manolopoulos. These authors have studied the same system using the CMD and the RPMD models. They considered two approximate approaches:

(1) Calculate the Kubo-transformed version of $F_{s}(\kappa, t)$-the incoherent relaxation function $\tilde{F}_{s}(\kappa, t)$, i.e., Eq. (2.24)—and then obtain the incoherent dynamic structure factor $S_{\text {inc }}(\boldsymbol{\kappa}, \omega)$ from its spectrum via Eq. (1.2); we refer to this approach as "RPMD-kubo" for the RPMD.

(2) Calculate the Kubo-transformed velocity correlation function and follow the same procedure as the "vv-kubo" method proposed in Section IIB; we refer to this as "RPMD-vv-kubo" for the RPMD.

It has been shown ${ }^{72,78}$, for both the CMD and RPMD models, that approach (1) agrees well with approach (2) above in the regime of small $\kappa$; for large $\kappa$, however, the agreement between the two approaches above becomes poor, presumably because the operator $e^{-i \kappa \cdot \hat{r}_{i}}$ becomes more nonlinear.

To summarize the results contained in Fig. 2, all comparisons of the incoherent dynamic structure $S_{i n c}(\boldsymbol{\kappa}, \omega)$ among the three TGA/LSC-IVR methods (proposed in Section IIB), and with other models ${ }^{72,78}$, show that the LSC-IVR is a quite consistent method for approximating quantum 
mechanical correlation functions involving both the linear and nonlinear operators if the time scale of the correlation function is not too long.

\section{Spectral Moment Test}

In Fig. 3, the three lowest moments $\mu_{n}(\kappa)$ of $S_{\text {inc }}(\kappa, \omega)$ obtained from our calculations are plotted as a function of the momentum transfer parameter $\kappa$, compared with the exact results in Eqs. (2.20)-(2.22) and the RPMD results of Ref. ${ }^{78}$. The spectral moments from the CMD are not available in Ref. ${ }^{72}$, but expected to be similar to those given by RPMD. Since the analysis in Appendix B shows that the "inelastic-std", "vv-kubo" and "vv-std” versions of our TGA/LSC-IVR approach are expected to produce $\mu_{0}(\kappa)$ and $\mu_{1}(\kappa)$ exactly (also see Table 1 ), the slight disagreements with the exact values seen in Fig. 3 are due to residual numerical error. Fig. 3 implies that the "inelastic-std" version of the TGA-LSC-IVR deviates more than the other two versions; we attribute this to the fact that the estimator for $F_{S}^{T G A / L S C-I V R}(t)$ in the Monte Carlo evaluation, Eq. (A.3), has more numerical cancellation from the phase term.

For the $2^{\text {nd }}$-order moment $\mu_{2}(\kappa)$, the results of the methods based on the Gaussian approximation, Eq. (2.10), are independent of $\kappa$ and only depend on how accurate the average kinetic energy $\left\langle\frac{\hat{\mathbf{p}}^{2}}{2 m N}\right\rangle$ is given by the velocity correlation function (see Eq. (2.22)). For example, the "vv-kubo" version of the TGA/LSC-IVR gives a value of $~ 65.0 \mathrm{~K}$ (for the present simulation of 108 para $-\mathrm{H}_{2}$ molecules per cell with periodic boundary condition ${ }^{26}$ ), and the accurate result by the imaginary time path integral Monte Carlo (PIMC) is $63.2 \mathrm{~K}^{48}$, so that the approximation to $\mu_{2}(\kappa)$ 
overestimates the result by less than $2.85 \%$. For comparison, it was reported in Ref. ${ }^{78}$ that the "RPMD-vv-kubo" result for $\mu_{2}(\kappa)$ exceeds the exact value by $6.3 \%$. Furthermore, the relative error in $\mu_{2}(\kappa)$ by the "inelastic-std" version of the TGA/LSC-IVR or by the "RPMD-kubo" method certainly depends on the momentum transfer parameter $\kappa$; these results are plotted in Fig. $4 . \quad$ It is encouraging to see that the relative error given by the "inelastic-std" TGA/LSC-IVR method is quite small even at large values of $\kappa$. For instance, at the largest $\kappa$ in Fig. 4, the relative error is about $8 \%$ while that given by the "RPMD-kubo"78 is over 524\%. In the regime where $\kappa$ is very small, the "inelastic-std" version of the TGA/LSC-IVR doesn't work as well, e.g., for the smallest value of $\kappa$ in the simulation, the relative error is about $18 \%$, which is close to that given by the "RPMD-kubo" in Ref. ${ }^{78}$ (about 19\%).

Shown in Fig. 5 are the first two even moments of $\tilde{S}_{\text {inc }}(\kappa, \omega)$ obtained from these three versions of the TGA/LSC-IVR, and from the two versions of the RPMD, in addition to the exact results in Eqs. (2.27) and (2.28). Since the "RPMD-kubo" directly calculates $\tilde{F}_{s}(\kappa, t)$ and then its spectrum $\tilde{S}_{\text {inc }}(\boldsymbol{\kappa}, \omega)$, it can be shown that the RPMD gives the exact results for $\underline{\mu}_{0}(\kappa)$ and $\underline{\mu}_{2}(\kappa)^{78}$. Fig. 5 demonstrates that all methods (the "vv-kubo" and the "vv-std" of the TGA/LSC-IVR and the "RPMD-vv-kubo") which are based the Gaussian approximation, Eq. (2.10), are a very good approximation for this system, and the "inelastic-std" also works well for the test of this set of moments ${ }^{119}$. 
Again, the overall comparisons of spectral moments as shown in Figs. 3-5 demonstrate that the LSC-IVR is a consistently good short-time approximation to time correlation functions involving both linear and nonlinear operators.

\section{Comparison with Experimental Data}

Though experimental data reported so far on pure liquid para-hydrogen around $14 \mathrm{~K}$ are not yet sufficient to compare with the incoherent dynamical structure factor $S_{\text {inc }}(\mathbf{\kappa}, \omega)$ for the whole range of the momentum transfer parameter $\kappa$ shown in Fig. 2, some inelastic neutron scattering experiments such as Ref. ${ }^{100,101}$ do provide experimental results on one or two points of $S_{\text {inc }}(\boldsymbol{\kappa}, \omega)$ for each $\kappa$. Very close to the system that we consider in this paper, pure liquid para-hydrogen at the state point $T=14.1(1) \mathrm{K}, v=22.95(3) \mathrm{nm}^{-3}$ has been examined in recent experiments by Colognesi et al. ${ }^{101}$. In addition to Section IIIA, Appendix D gives more details on the simulation of the experiment, and Fig. 6 shows the momentum transfer parameter $\kappa_{F}(\omega)$ or $\kappa_{B}(\omega)$ as a function of the energy transfer parameter $\omega$ for the forward or backward scattering in the experiment.

All available results of the incoherent dynamic structure factor $S(\kappa(\omega), \omega)$ computed by all three versions - the "inelastic-std", the "vv-kubo" and the "vv-std"—of the TGA/LSC-IVR formulation of the time correlation function are plotted together with the experimental data in Fig. 7. Panel (a) shows the comparison for the forward scattering along the $\kappa_{F}(\omega)$ in Fig. 6, which represents the momentum transfer from $\kappa=1.780$ to $3.716 \AA^{-1}$, i.e., in the intermediate regime 
between the diffusive and the impulsive regime (See Fig. 2 and Appendix C). Overall, the three versions of the TGA/LSC-IVR agree quite well with the experimental results. There is some discrepancy among the three versions near the peak, $\kappa \approx 2.18 \AA^{-1}$, with the "vv-kubo" version seeming to give the best agreement with experiment. Panel (b) shows the comparison for the backward scattering along $\kappa_{B}(\omega)$ in Fig. 6, which samples the momentum transfer from $\kappa=3.532$ to $5.551 \AA^{-1}$, i.e., from the intermediate to the impulsive regime (See Fig. 2 and Appendix C). Again, all three versions of the TGA/LSC-IVR give reasonably good agreement with the experimental data, though there is somewhat more disagreement among the three versions in the backward scattering case. The "inelastic-std" agrees best with the experiment for the regime $-5 \mathrm{meV} \leq \omega \leq 5 \mathrm{meV}$ or $3.532 \AA^{-1} \leq \kappa(\omega) \leq 4.343 \AA^{-1}$, while the "vv-kubo" does so for larger $\omega$ or $\kappa(\omega)$. Note that the discrepancies in Fig. 7 could be due to various factors, including the TGA introduced to obtain the analytical form for the Wigner function $A_{w}^{\beta}$, i.e., Eq (2.14), or the over-simplified isotropic pair potential (the SG model ${ }^{116}$ ) used in a process involved with the rotational excitation from $J=0$ to $J=1^{101}$ (not good for the spherical approximation). And we notice that the system size may have its effect on the simulation results (at least in the diffusive region) ${ }^{80}$, but currently our simulations are limited up to 216 molecules per box (see Appendix D). How the results can be extrapolated to infinite system size is of course worth investigating in future.

We note that the "RPMD-vv-kubo" approach also gives very good agreement with these experiments, though the "RPMD-kubo" is believed to give poor results ${ }^{78}$ (Since the "RPMD-kubo" 
results are not available in Ref. ${ }^{78}$, we don’t systematically compare the LSC-IVR and the RPMD results here). The CMD model shares the same behavior as the RPMD, as seen in the simulations for some similar experiments ${ }^{100,120}$. These results obtained through the Kubo-transformed velocity correlation function based on the RPMD and the CMD, in addition to what is shown in Fig. 7 for the TGA/LSC-IVR, verify that the Gaussian approximation, Eq. (2.10), is very good for calculating the incoherent dynamic structure factor of liquid para-hydrogen even at $T=14 \mathrm{~K}$, which indicates that the non-Maxwellian part of the velocity distribution is negligible (consistent with the conclusion in Appendix C based on Fig. 2).

In summary, it is clear from Fig. 7 that all three methods proposed in Section IIB based on the TGA/LSC-IVR give reasonably good agreement with one another and a semi-quantitative description of both the forward and backward scattering data. This agrees with our previous comments in Section IIIB and IIIC that the LSC-IVR formulation of time correlation functions treats both the linear and nonlinear operators in a fairly consistent manner in such a realistic highly anharmonic system.

\section{Conclusions}

In this paper, we have presented the first systematic examination of the consistency of the LSC-IVR approximation for time correlation functions with different operators for a realistic model of a complex system far from the harmonic regime. We applied the TGA/LSC-IVR approximation to include quantum dynamical effects in the simulation of the inelastic neutron scattering from liquid 
para-hydrogen at $T=14 \mathrm{~K}$. Taking advantage of the fact the velocity distribution is still very nearly Gaussian even for such a low-temperature liquid system, we were able to calculate the incoherent dynamic structure factor $S_{\text {inc }}(\kappa, \omega)$ directly by using the self-part of the intermediate scattering function, Eq. (2.6), or indirectly by implementing the Gaussian approximation, Eq. (2.10), based on velocity correlation functions (both the standard and the Kubo-transformed versions). These approaches based on the TGA/LSC-IVR all give semi-quantitative agreement with inelastic neutron scattering experiments ${ }^{101}$. Together with the spectral moment tests, it clearly demonstrates that the LSC-IVR is a good short-time approximation to the quantum dynamical time correlation function and can treat different operators (both the linear and the nonlinear operators) fairly consistently.

For dynamical processes in condensed phase systems where quantum mechanics play a significant role and time scales of correlation functions are usually not very long, the consistency of the LSC-IVR in treating different operators makes it a practical and versatile method for studying these phenomena semi-quantitatively. It will be interesting to apply the LSC-IVR to complex systems at even lower temperature (such as normal and superfluid liquid He) where quantum effects are more pronounced, to see how well the Gaussian approximation (Eq. (2.10)) works ${ }^{52,121}$, and its comparison with the direct calculation of the incoherent dynamic structure factor using Eq. (2.6) and also with experimental data ${ }^{121-126}$. It will also be an interesting task to use the LSC-IVR to calculate the coherent dynamic structure factor, which reflects the collective behavior of liquids rather than the 
single-particle motion ${ }^{72,95,104,127}$, as demonstrated in experiments on liquid $\mathrm{H}_{2} / \mathrm{D}_{2}{ }^{127-130}$ and liquid $\mathrm{He}^{131}$.

However, we did observe some inconsistencies among the different versions of the LSC-IVR approach in the long time behavior of the time correlation function. Recently we have derived a different method $^{9}$ to improve the long-time dynamical behavior of the LSC-IVR without having to deal with the phase cancellation problems in the full version of the SC-IVR. This method with its modified classical dynamics can in principle guarantee that the distribution generated for the operator $\hat{A}^{\beta}$ is invariant with time for the case $\hat{A}=1$ (i.e., $\hat{A}^{\beta}=\frac{1}{Z} e^{-\beta \hat{H}}$, the Boltzmann operator itself), which remedies one of the principle defects of the LSC-IVR. It will be interestingly in future work to apply this improved version of the $\operatorname{LSC} \operatorname{IVR}^{9}$ and other more advanced SC-IVRs ${ }^{22,57,58}$ to complex (large) systems. For instance, it would be natural to use the Fourier transform forward-backward IVR (FB-IVR) approach ${ }^{22,57}$ to calculate $F_{s}(\boldsymbol{\kappa}, t)$, Eq. (2.6), by introducing the momentum jump $\hbar \kappa$ at time $t$ between the forward trajectory $(0 \rightarrow t)$ and its backward counterpart $(t \rightarrow 0)$, similar to an early study on incoherent neutron scattering from solid $\mathrm{HCN}^{132}$.

Finally, we note that using the $\mathrm{RPMD}^{76}$ results as the prior in the MEAC ${ }^{84-89}$ approach (the RPMD+MEAC) recently suggested by Manolopoulos et $a .^{81}$, (though in an earlier proposed CMD+MEAC ${ }^{88}$ paper, the author mentioned about the possibility to use the CMD results as the prior in the MEAC, but no further work was shown), could in practice improve the behavior of the RPMD model to calculate the time correlation function with nonlinear operators. E.g., the RPMD+MEAC 
could probably reduce the large relative error of the "RPMD-kubo" approach for large $\kappa^{133}$ in Figs.

3-4. Quite interestingly, Ref. ${ }^{81}$ shows that even classical dynamics combined with the MEAC (the CD+MEAC) could produce similar results as those given by the RPMD+MEAC. Since the LSC-IVR is a consistently better approximation to the quantum mechanical correlation function than classical dynamics, the LSC-IVR combined with the MEAC (the LSC-IVR+MEAC) could very likely improve the long-time behavior of the original LSC-IVR. A further investigation of this extension of the LSC-IVR approach for treating quantum dynamical phenomena in large molecular systems is certainly warranted.

\section{Acknowledgement}

We thank D. Manolopoulos for providing the RPMD results ${ }^{78}$ and the experiment data ${ }^{101}$ and for some useful discussions. This work was supported by the Office of Naval Research Grant No. N00014-05-1-0457 and by the Director, Office of Science, Office of Basic Energy Sciences, Chemical Sciences, Geosciences, and Biosciences Division, U.S. Department of Energy under Contract No. DE-AC02-05CH11231. We also acknowledge a generous allocation of supercomputing time from the National Energy Research Scientific Computing Center (NERSC). 


\section{Appendix A: TGA/LSC-IVR Formulation of the Intermediate Scattering Function}

The TGA/LSC-IVR formulation of the intermediate scattering function $F(\boldsymbol{\kappa}, t)$, Eq. (2.2), or its self-part $F_{S}(\boldsymbol{\kappa}, t)$, Eq. (2.6), is related to the operator $\hat{A}=\frac{1}{N} \sum_{i=1}^{N} e^{-i \boldsymbol{k} \cdot \hat{\mathbf{x}}_{i}}$ or $\hat{A}^{\beta}=\frac{1}{Z} e^{-\beta \hat{H}}\left(\frac{1}{N} \sum_{i=1}^{N} e^{-i \boldsymbol{\kappa} \hat{\mathbf{x}}_{i}}\right)$, the Fourier transform of which is

$$
\begin{aligned}
A_{w}^{\beta}\left(\mathbf{x}_{0}, \mathbf{p}_{0}\right)= & \int d \boldsymbol{\Delta} \mathbf{x}\left\langle\mathbf{x}_{0}-\Delta \mathbf{x} / 2\left|\hat{A}^{\beta}\right| \mathbf{x}_{0}+\Delta \mathbf{x} / 2\right\rangle e^{i \mathbf{p}_{0}^{T} \Delta \mathbf{x} / \hbar} \\
= & \frac{1}{N Z} \int d \mathbf{q} \int d \boldsymbol{\Delta} \mathbf{x}\left\langle\mathbf{x}_{0}-\Delta \mathbf{x} / 2\left|e^{-\beta \hat{H} / 2}\right| \mathbf{q}\right\rangle \\
& \cdot\left\langle\mathbf{q}\left|e^{-\beta \hat{H} / 2} \sum_{i=1}^{N} e^{-i \mathbf{k} \cdot \hat{\mathbf{x}}_{i}}\right| \mathbf{x}_{0}+\Delta \mathbf{x} / 2\right\rangle e^{i \mathbf{p}_{0}^{T} \mathbf{\Delta x} / \hbar} \\
\cong & \frac{1}{Z} \int d \mathbf{q}\left(\frac{1}{2 \pi}\right)^{3 N} \frac{\exp \left(2 \gamma\left(\frac{\beta}{2}\right)\right)}{|\operatorname{det}(\mathbf{G}(\tau))|^{1 / 2}} \\
& \cdot \exp \left(-\left(\mathbf{x}_{0}-\mathbf{q}\left(\frac{\beta}{2}\right)\right)^{T} \mathbf{G}^{-1}\left(\frac{\beta}{2}\right)\left(\mathbf{x}_{0}-\mathbf{q}\left(\frac{\beta}{2}\right)\right)\right) \\
& \cdot \int d \boldsymbol{\Delta} \mathbf{x} \exp \left(-\frac{1}{4} \Delta \mathbf{x}^{T} \mathbf{G}^{-1}\left(\frac{\beta}{2}\right) \Delta \mathbf{x}\right) \frac{1}{N} \sum_{i=1}^{N} e^{-i \mathbf{k} \cdot\left(\mathbf{x}_{i}-\Delta \mathbf{x}_{i}\right)} e^{i \mathbf{p}_{0}^{T} \mathbf{\Delta x} / \hbar}
\end{aligned}
$$

The integral over $\boldsymbol{\Delta x}$ gives Eqs. (2.14) and (2.17). Substituting them into Eq. (2.11), one obtains the expression of the correlation function as

$$
\begin{aligned}
C_{A B}^{T G A / L S C-I V R}(t)= & \frac{1}{Z} \int d \mathbf{q}_{0} \frac{1}{(4 \pi)^{3 N / 2}} \frac{\exp \left(2 \gamma\left(\frac{\beta}{2}\right)\right)}{\left|\operatorname{det} \mathbf{G}\left(\frac{\beta}{2}\right)\right|^{1 / 2}} \\
& \cdot \int d \mathbf{x}_{0} \frac{1}{\pi^{3 N / 2}\left|\operatorname{det} \mathbf{G}\left(\frac{\beta}{2}\right)\right|^{1 / 2}} \exp \left(-\left(\mathbf{x}_{0}-\mathbf{q}\left(\frac{\beta}{2}\right)\right)^{T} \mathbf{G}^{-1}\left(\frac{\beta}{2}\right)\left(\mathbf{x}_{0}-\mathbf{q}\left(\frac{\beta}{2}\right)\right)\right) \\
& \cdot \int d \mathbf{p}_{0} \frac{\left|\operatorname{det} \mathbf{G}\left(\frac{\beta}{2}\right)\right|^{1 / 2}}{\left(\pi \hbar^{2}\right)^{3 N / 2}} \exp \left(-\mathbf{p}_{0}^{T} \mathbf{G}\left(\frac{\beta}{2}\right) \mathbf{p}_{0} / \hbar^{2}\right) \\
& \cdot g_{A B}^{T G A / L S C-I V R}\left(\mathbf{x}_{0}, \mathbf{p}_{0}, \mathbf{q}\left(\frac{\beta}{2}\right) ; t\right)
\end{aligned}
$$

where the estimator (in the Monte Carlo evaluation of Eq. (A.2), which is described in Section IV of Ref. $^{32}$ ) is given by 


$$
\begin{aligned}
g_{A B}^{T G A / L S C-I V R}\left(\mathbf{x}_{0}, \mathbf{p}_{0}, \mathbf{q}\left(\frac{\beta}{2}\right) ; t\right)=f_{A B}^{T G A / L S C-I V R}\left(\mathbf{x}_{0}, \mathbf{p}_{0}, \mathbf{q}\left(\frac{\beta}{2}\right)\right) B_{w}\left(\mathbf{x}_{t}, \mathbf{p}_{t}\right) \\
=\frac{1}{N} \sum_{i=1}^{N} e^{i \boldsymbol{\kappa} \cdot \mathbf{x}_{i}(t)} e^{-i \boldsymbol{\kappa} \cdot \mathbf{x}_{i}(0)} \exp \left[\mathbf{p}_{0}^{T} \cdot \mathbf{G}_{\mathbf{x}_{i}}\left(\frac{\beta}{2}\right) \cdot \mathbf{\kappa} / \hbar-\frac{1}{4} \boldsymbol{\kappa}^{T} \cdot \mathbf{G}_{\mathbf{x}_{i} \mathbf{x}_{i}}\left(\frac{\beta}{2}\right) \cdot \mathbf{\kappa}\right] \\
\quad \text { for } F_{S}(\mathbf{\kappa}, t) \\
=\frac{1}{N} \sum_{j=1}^{N} e^{i \boldsymbol{\kappa} \cdot \mathbf{x}_{j}(t)} \sum_{i=1}^{N} e^{-i \boldsymbol{\kappa} \cdot \mathbf{x}_{i}(0)} \exp \left[\mathbf{p}_{0}^{T} \cdot \mathbf{G}_{\mathbf{x}_{i}}\left(\frac{\beta}{2}\right) \cdot \boldsymbol{\kappa} / \hbar-\frac{1}{4} \boldsymbol{\kappa}^{T} \cdot \mathbf{G}_{\mathbf{x}_{i} \mathbf{x}_{i}}\left(\frac{\beta}{2}\right) \cdot \mathbf{\kappa}\right] \\
\quad \text { for } F(\mathbf{\kappa}, t)
\end{aligned}
$$

For the isotropic system, both $F(\boldsymbol{\kappa}, t)$ and $F_{S}(\kappa, t)$ depend only on $\kappa=|\kappa|$. Since the direction of the vector $\boldsymbol{\kappa}$ doesn't matter, for convenience one can choose it along the $x$-axis in the calculation, i.e., $\hat{A}=\frac{1}{N} \sum_{i=1}^{N} e^{-i \kappa \cdot \hat{\mathbf{x}}_{i}}=\frac{1}{N} \sum_{i=1}^{N} e^{-i \kappa \hat{x}_{i}}$, where $\hat{x}_{i}$ is the $x$-axis component of the position operator $\hat{\mathbf{x}}_{i}$ for the $i$-th particle, and Eq. (A.3) can be simplified further. Better statistics could be obtained by averaging over the direction of $\boldsymbol{\kappa}$ over the three Cartesian axes.

\section{Appendix B: Analytical Analysis of Spectral Moments}

The two lowest spectral moments in Eq. (2.19) are given exactly by the LSC-IVR. Based on the well-known identity for the trace of a product of any two operators $\hat{P}$ and $\hat{Q}$,

$$
\operatorname{Tr}(\hat{P} \hat{Q})=(2 \pi \hbar)^{-3 N} \int d \mathbf{x} \int d \mathbf{p} P_{w}(\mathbf{x}, \mathbf{p}) Q_{w}(\mathbf{x}, \mathbf{p})
$$

and the relation

$$
\mu_{0}(\kappa)=F_{S}(\kappa, t=0)
$$

it immediately follows that the LSC-IVR, Eq. (2.11), gives the exact result for $\mu_{0}(\kappa)$. From the LSC-IVR expression for $F_{S}(\kappa, t)$, 


$$
\begin{aligned}
F_{S}^{L S C-I V R}(\boldsymbol{\kappa}, t)= & (2 \pi \hbar)^{-3 N}(N Z)^{-1} \int d \mathbf{x}_{0} \int d \mathbf{p}_{0} \int d \Delta \mathbf{x} \\
& \left\langle\mathbf{x}_{0}-\Delta \mathbf{x} / 2\left|e^{-\beta \hat{H}} \sum_{i=1}^{N} e^{-i \boldsymbol{\kappa} \cdot \hat{\mathbf{x}}_{i}}\right| \mathbf{x}_{0}+\Delta \mathbf{x} / 2\right\rangle e^{i \mathbf{p}_{0}^{T} \Delta \mathbf{x} / \hbar} e^{i \boldsymbol{\kappa} \cdot \mathbf{x}_{i}(t)} \\
= & (2 \pi \hbar)^{-3 N}(N Z)^{-1} \int d \mathbf{x}_{0} \int d \mathbf{p}_{0} \int d \Delta \mathbf{x} \\
& \left\langle\mathbf{x}_{0}-\Delta \mathbf{x} / 2\left|e^{-\beta \hat{H}}\right| \mathbf{x}_{0}+\Delta \mathbf{x} / 2\right\rangle \sum_{i=1}^{N} e^{-i \boldsymbol{\kappa} \cdot\left(\mathbf{x}_{i}(0)-\mathbf{x}_{i}(t)\right)} e^{i \mathbf{p}_{0}^{T} \Delta \mathbf{x} / \hbar-i \mathbf{k} \cdot \mathbf{x}_{i} / 2}
\end{aligned}
$$

one can show that

$$
\begin{aligned}
\frac{d}{d t} F_{S}^{L S C-I V R}(\boldsymbol{\kappa}, t=0)= & i(2 \pi \hbar)^{-3 N}(N Z)^{-1} \sum_{i=1}^{N} \int d \mathbf{x}_{0} \int d \mathbf{p}_{0} \int d \Delta \mathbf{x} \\
& \left\langle\mathbf{x}_{0}-\Delta \mathbf{x} / 2\left|e^{-\beta \hat{H}}\right| \mathbf{x}_{0}+\Delta \mathbf{x} / 2\right\rangle e^{i \mathbf{p}_{0}^{T} \Delta \mathbf{x} / \hbar-i \boldsymbol{k} \cdot \Delta \mathbf{x}_{i} / 2} \frac{\boldsymbol{\kappa} \cdot \mathbf{p}_{i}(0)}{m}
\end{aligned}
$$

In each term of the sum, if one replaces the $i$-th particle component of the variable $\mathbf{p}_{0}$ by $\mathbf{p}_{i}(0) \rightarrow \mathbf{p}_{i}(0)+\hbar \mathbf{\kappa} / 2$ (which does affect the integral over $\left.\mathbf{p}_{0}\right)$ and takes advantage of the symmetry of the matrix element

$$
\left\langle\mathbf{x}_{0}-\Delta \mathbf{x} / 2\left|e^{-\beta \hat{H}}\right| \mathbf{x}_{0}+\Delta \mathbf{x} / 2\right\rangle=\left\langle\mathbf{x}_{0}+\Delta \mathbf{x} / 2\left|e^{-\beta \hat{H}}\right| \mathbf{x}_{0}-\Delta \mathbf{x} / 2\right\rangle
$$

then Eq. (B.4) becomes

$$
\frac{d}{d t} F_{S}^{L S C-I V R}(\kappa, t=0)=\frac{i \hbar \kappa^{2}}{2 m} \equiv i \omega_{R},
$$

so that

$$
\mu_{1}=i^{-1} \frac{d}{d t}\left[e^{-i \omega_{R} t} F_{s}(\kappa, t)\right]_{t=0}=0
$$

Interestingly, the LSC-IVR is still exact $\mu_{0}(\kappa)$ and $\mu_{1}(\kappa)$ when using the TGA. In fact, it is straightforward to show the TGA/LSC-IVR expression for $F_{S}(\kappa, t)$, Eq. (A.2) with Eq. (A.3), gives the exact value at $t=0$, i.e.,

$$
F_{S}^{T G A / L S C-I V R}(\kappa)=1
$$


which is the same as Eq. (2.20). Also note that the proof above for $\mu_{1}(\kappa)$ in the LSC-IVR only requires the symmetry of the Boltzmann matrix element, i.e., Eq. (B.5), which is certainly true for the TGA (see Eq. (3.6) of Ref. ${ }^{32}$ and its discussion), so that it then follows that the TGA/LSC-IVR expression of $F_{S}(\kappa, t)$ also satisfies Eq. (B.7), i.e., gives the exact value of $\mu_{1}(\kappa)$.

\section{Appendix C: Incoherent Dynamic Structure in the Limit of Large and Small $\kappa$}

The results for $S_{\text {inc }}(\boldsymbol{\kappa}, \omega)$ in Fig. 2 tend to be Gaussian for all three versions of the LSC-IV for large $\kappa$. This indicates that even for such a low-temperature system as liquid para-hydrogen at $T=14 \mathrm{~K}$, the velocity distribution is still nearly Gaussian. To see this more clearly, substitute Eq. (2.7) into Eq. (2.1), make a change of variable $y=\kappa t$, and expand the $\gamma_{n}$ functions in Eq. (2.8) and (2.9) etc. in powers of $y$, giving

$$
S_{i n c}(\boldsymbol{\kappa}, \omega)=\frac{1}{2 \pi \kappa} \int_{-\infty}^{\infty} \exp \left[-i y\left(\omega-\omega_{R}\right) / \kappa\right] \exp \left[\sum_{n=1}^{\infty} g_{n}(y)\right] d y,
$$

where

$$
\begin{aligned}
& g_{1}(y)=-\left\langle\hat{v}_{\kappa}^{2}\right\rangle y^{2} / 2+O(1 / \kappa) \\
& g_{2}(y)=\left[\left\langle\hat{v}_{\kappa}^{4}\right\rangle-3\left\langle\hat{v}_{\kappa}^{2}\right\rangle^{2}\right] y^{4} / 24+O(1 / \kappa)
\end{aligned}
$$

and so on ${ }^{102}$. As $\kappa \rightarrow \infty$ (in the impulsive regime), if the velocity distribution is Gaussian, $g_{2}(y)$ and higher order terms vanish, which leads to

$$
\lim _{\kappa \rightarrow \infty} S_{\text {inc }}(\boldsymbol{\kappa}, \omega)=\left(\frac{\beta_{\text {eff }} m}{2 \pi \kappa^{2}}\right)^{1 / 2} \exp \left[-\beta_{\text {eff }} m\left(\omega-\omega_{R}\right)^{2} / 2 \kappa^{2}\right]
$$

with $\beta_{\text {eff }}=\frac{3 N}{2\left\langle\hat{\mathbf{p}}^{2} / 2 m\right\rangle}$, for isotropic systems ${ }^{134,135}$. 
Fig. 2 verifies that the non-Maxwellian effect of the velocity distribution for such a low-temperature system as liquid para-hydrogen at $T=14 \mathrm{~K}$ is still negligible, which is why Eqs. (2.10) is indeed a good approximation for all $\kappa$, allowing one to use the velocity correlation function to calculate the incoherent dynamic structure factor for this system as pointed out in Section IIA. The "inelastic-std", "vv-kubo" and "vv-std" methods proposed in Section IIB thus would give essentially the same results for $S_{i n c}(\boldsymbol{\kappa}, \omega)$ if the quantum mechanical correlation functions were exactly calculated. Comparison of $S_{i n c}(\boldsymbol{\kappa}, \omega)$ based on the three approaches does shed light on the consistency of the LSC-IVR approximation to deal with different operators.

However, for very small $\kappa$ (in the diffusive regime), the incoherent dynamic structure $S_{\text {inc }}(\boldsymbol{\kappa}, \omega)$ turns out to be Lorentzian instead. Since the time scale of the correlation function $F_{s}(\kappa, t)$ is very long, as shown in Fig.1 (longer than that of $C_{\mathbf{v v}}\left(t^{\prime}\right)$ ), Eq. (2.10) is equivalent to

$$
F_{s}(\kappa, t)=\exp \left[\left(i \omega_{R}-\kappa^{2} D\right) t\right]
$$

where $D$ is the diffusion constant. As a consequence, one has a Lorentzian-like spectrum in the very small $\kappa$ region $^{104}$, i.e.,

$$
\lim _{\kappa \rightarrow 0} S_{i n c}(\boldsymbol{\kappa}, \omega)=\frac{D \kappa^{2} / \pi}{\omega^{2}+\left(D \kappa^{2}\right)^{2}}
$$

as seen in Fig. 2.

\section{Appendix D: Additional Simulation Details on the Inelastic Neutron Scattering Experiment}

In the experiment ${ }^{101}$ that we consider in Section IIID, the neutron scattering 
spectrometer - the TOSCA-II apparatus ${ }^{136,137}$ — was used to scatter neutrons from the liquid at a forward and a backward angle (i.e., the angle between the initial and the final wave vectors of the neutron, $\boldsymbol{\kappa}_{i}$ and $\boldsymbol{\kappa}_{f}$ in Eqs. (2.3) and (2.4)). The forward angle is $42.6^{\circ}$ and the scattered neutron is at energy of $3.35 \mathrm{meV}$ (i.e., $\frac{\hbar^{2} \boldsymbol{\kappa}_{f}^{2}}{2 m_{n}}$ in Eq. (2.4)), and the quantities for the backward direction are $137.7^{\circ}$ and $3.32 \mathrm{meV}$ respectively. Since the experiment ${ }^{101}$ actually measured the cross section for the inelastic scattering process involved with the rotational excitation $n+\mathrm{H}_{2}(J=0) \rightarrow n+\mathrm{H}_{2}(J=1)$, the energy transfer $\hbar \omega$ should be replaced by $\hbar\left(\omega+\omega_{10}\right)$ in Eq. (2.4), i.e.,

$$
\hbar\left(\omega+\omega_{10}\right)=\frac{\hbar^{2}\left(\boldsymbol{\kappa}_{i}^{2}-\boldsymbol{\kappa}_{f}^{2}\right)}{2 m_{n}},
$$

where $\hbar \omega_{10}=14.53 \mathrm{meV}$ is the excitation energy from the rotational ground state ( $J=0$ ) to its first excited state $(J=1)$ of $\mathrm{H}_{2}$. By virtue of the conservation laws, i.e., Eqs. (2.3) and (D.1), it is straightforward to calculate the momentum transfer parameter $\kappa_{F}(\omega)$ or $\kappa_{B}(\omega)$ as a function of the energy transfer parameter $\omega$ for the forward or backward scattering experiments. Both $\kappa_{F}(\omega)$ and $\kappa_{B}(\omega)$ are plotted in Fig. 6 in the range of $\omega$ where the experiment ${ }^{101}$ detects $S_{\text {inc }}(\kappa(\omega), \omega)$. Such two kinematic lines for a wider range of $\omega$ are shown in Fig. 1 of Ref. ${ }^{101}$.

Because of the relation, Eq. (3.1), in the simulation using a finite cell with periodic boundary conditions, the available momentum transfer parameter $\kappa(\omega)$ depends on the size of the simulation box. For a particular box size, only a few points of $\kappa_{F}(\omega)$ or $\kappa_{B}(\omega)$ in Fig. 6 satisfy Eq. (3.1) in the range of experimental data $(-5 \mathrm{meV} \leq \omega \leq 25 \mathrm{meV})$. To obtain more computational results, four 
sets of simulations are carried out: 64, 125, and 216 molecules in a box starting from a cubic lattice, and also 108 molecules in a box with the face-centered cubic structure as the initial configuration.

The TOSCA-II experimental data ${ }^{101}$ along the two kinematic lines in the $(\kappa, \omega)$ plane in Fig. 6 provide only two points of $S_{\text {inc }}(\kappa, \omega)$ for each $\kappa$ in the overlapped regime of $\kappa_{F}$ and $\kappa_{B}$, i.e., $3.532 \AA^{-1} \leq \kappa \leq 3.716 \AA^{-1}$, and but one point of $S_{\text {inc }}(\kappa, \omega)$ for each $\kappa$ in other regimes. More favorable experimental results will of course be those providing the whole spectrum $S_{\text {inc }}(\kappa, \omega)$ for each $\kappa$ to allow one to have a direct comparison to Fig. 2, which would give much more information to check the theoretical simulations. Nevertheless, the TOSCA-II experimental data ${ }^{101}$ still provide a useful test for the accuracy of the TGA/LSC-IVR approximation while exact quantum mechanical results are not available.

Fig. 7 shows the incoherent dynamic structure $S(\kappa(\omega), \omega)$ calculated from the three methods proposed in Section IIB based on the TGA/LSC-IVR, compared with the experimental data. Since the TOSCA backward scattering data contain "a possible spurious background"101, an additional linear polynomial $\left(4 \times 10^{-5} \mathrm{meV}^{-2}\right) \times \omega$ is added to the simulated $S_{\text {inc }}(\kappa(\omega), \omega)$ in Panel (b) as suggested by Ref. ${ }^{78,101}$. This is not necessary for Panel (a) since the background has already been removed from experimental forward scattering data ${ }^{78,101}$. 


\section{References}

$1 \quad$ B. J. Berne and G. D. Harp, Adv. Chem. Phys. 17, 63 (1970).

W. H. Miller, S. D. Schwartz, and J. W. Tromp, J. Chem. Phys. 79 (10), 4889 (1983).

R. Kubo, M. Toda, and N. Hashitsume, Statistical Physics II: Nonequilibrium Statistical Mechanics, 2nd ed. (Springer-Verlag, Heidelberg, 1991).

W. H. Miller, J. Chem. Phys. 53, 3578 (1970).

W. H. Miller, J. Phys. Chem. A 105 (13), 2942 (2001).

M. Thoss and H. B. Wang, Ann. Rev. Phys. Chem. 55, 299 (2004).

W. H. Miller, Proc. Nat. Acad. Sci. USA. 102 (19), 6660 (2005).

W. H. Miller, J. Chem. Phys. 125 (13), 132305 (2006).

J. Liu and W. H. Miller, J. Chem. Phys. 126, 234110 (2007).

M. F. Herman and E. Kluk, Chem. Phys. 91, 27 (1984).

E. J. Heller, J. Chem. Phys. 95 (12), 9431 (1991).

J. Cao and G. A. Voth, J. Chem. Phys. 104 (1), 273 (1996). 
S. Zhang and E. Pollak, J. Chem. Phys. 119, 11058 (2003).

W. H. Miller, Adv. Chem. Phys. 25, 69 (1974).

W. H. Miller, Adv. Chem. Phys. 30, 77 (1975).

W. H. Miller, Faraday Discuss. (110), 1 (1998).

M. A. Sepulveda and F. Grossmann, Adv. Chem. Phys. XCVI, 191 (1996).

D. J. Tannor and S. Garashchuk, Annu. Rev. Phys. Chem. 51, 553 (2000).

N. Ananth, C. Venkataraman, and W. H. Miller, J. Chem. Phys. 127 (8) (2007).

J. Liu and W. H. Miller, J. Chem. Phys. 127, 114506 (2007).

B. B. Issack and P. N. Roy, J. Chem. Phys. 126 (2) (2007).

X. Sun and W. H. Miller, J. Chem. Phys. 106 (3), 916 (1997).

H. Wang, X. Sun, and W. H. Miller, J. Chem. Phys. 108 (23), 9726 (1998).

X. Sun, H. Wang, and W. H. Miller, J. Chem. Phys. 109 (17), 7064 (1998).

T. Yamamoto, H. B. Wang, and W. H. Miller, J. Chem. Phys. 116 (17), 7335 (2002).

J. Liu and W. H. Miller, J. Chem. Phys. 125 (22), 224104 (2006).

R. Hernandez and G. A. Voth, Chem. Phys. 233 (2-3), 243 (1998).

Q. Shi and E. Geva, J. Phys. Chem. A 107, 9059 (2003).

J. A. Poulsen, G. Nyman, and P. J. Rossky, J. Chem. Phys. 119 (23), 12179 (2003).

E. P. Wigner, Phys. Rev. 40, 749 (1932). 
E. J. Wigner, Trans. Faraday Soc. 34, 29 (1938).

E. J. Heller, J. Chem. Phys. 65, 1289 (1976).

H. W. Lee and M. O. Scully, J. Chem. Phys. 73 (5), 2238 (1980).

E. Pollak and J. L. Liao, J. Chem. Phys. 108 (7), 2733 (1998).

Q. Shi and E. Geva, J. Chem. Phys. 118 (18), 8173 (2003).

N. Makri and K. Thompson, Chem. Phys. Lett. 291, 101 (1998).

K. Thompson and N. Makri, J. Chem. Phys. 110, 1343 (1999).

J. Shao and N. Makri, J. Phys. Chem. A 103, 7753 (1999).

J. Shao and N. Makri, J. Phys. Chem. A 103, 9479 (1999).

N. Makri, J. Phys. Chem. B 106 (33), 8390 (2002).

N. J. Wright and N. Makri, J. Chem. Phys. 119 (3), 1634 (2003).

A. Nakayama and N. Makri, J. Chem. Phys. 119, 8592 (2003).

N. J. Wright and N. Makri, J. Phys. Chem. B 108, 6816 (2004).

A. Nakayama and N. Makri, Chem. Phys. 304, 147 (2004).

N. Makri, A. Nakayama, and N. Wright, J. Theor. Comp. Chem. 3, 391 (2004).

A. Nakayama and N. Makri, Proc. Nat. Acad Sci. USA 102, 4230 (2005).

J. Liu and N. Makri, Chem. Phys. 322 (1-2), 23 (2006).

J. Liu, A. Nakayama, and N. Makri, Mol. Phys. 104 (8), 1267 (2006).

J. Kegerreis and N. Makri, J. Comput. Chem. 28 (4), 818 (2007). 
E. Bukhman and N. Makri, J. Phys. Chem. A 111 (44), 11320 (2007).

X. Sun and W. H. Miller, J. Chem. Phys. 110 (14), 6635 (1999).

M. Thoss, H. Wang, and W. H. Miller, J. Chem. Phys. 114 (21), 9220 (2001).

J. A. Poulsen, G. Nyman, and P. J. Rossky, J. Phys. Chem. B 108 (51), 19799 (2004).

J. A. Poulsen, G. Nyman, and P. J. Rossky, J. Phys. Chem. A 108 (41), 8743 (2004).

B. J. Ka, Q. Shi, and E. Geva, J. Phys. Chem. A 109 (25), 5527 (2005).

B. J. Ka and E. Geva, J. Phys. Chem. A 110 (31), 9555 (2006).

J. Cao and G. A. Voth, J. Chem. Phys. 99, 10070 (1993).

J. Cao and G. A. Voth, J. Chem. Phys. 101 (7), 6157 (1994).

J. Cao and G. A. Voth, J. Chem. Phys. 101 (7), 6168 (1994).

J. Cao and G. A. Voth, J. Chem. Phys. 100 (7), 5106 (1994).

J. Cao and G. A. Voth, J. Chem. Phys. 101, 6168 (1994).

J. Cao and G. A. Voth, J. Chem. Phys. 101, 6184 (1994).

S. Jang and G. A. Voth, J. Chem. Phys. 111, 2371 (1999).

S. Jang and G. A. Voth, J. Chem. Phys. 111 (6), 2357 (1999).

D. R. Reichman, P. N. Roy, S. Jang, and G. A. Voth, J. Chem. Phys. 113 (3), 919 (2000).

T. D. Hone and G. A. Voth, J. Chem. Phys. 121 (13), 6412 (2004).

F. Paesani, S. Luchi, and G. A. Voth, J. Chem. Phys. 127, 074506 (2007).

K. Kinugawa, Chem. Phys. Lett. 292, 454 (1998). 
K. Kinugawa, H. Nagao, and K. Ohta, J. Chem. Phys. 114, 1454 (2001).

I. R. Craig and D. E. Manolopoulos, J. Chem. Phys. 121 (8), 3368 (2004).

I. R. Craig and D. E. Manolopoulos, J. Chem. Phys. 122 (8) (2005).

I. R. Craig and D. E. Manolopoulos, Chem. Phys. 322 (1-2), 236 (2006).

T. F. Miller and D. E. Manolopoulos, J. Chem. Phys. 123 (15), 154504 (2005).

T. F. Miller and D. E. Manolopoulos, J. Chem. Phys. 122 (18), 184503 (2005).

S. Habershon, B. J. Braams, and D. E. Manolopoulos, J. Chem. Phys. 127, 174108 (2007).

Q. Shi and E. Geva, J. Chem. Phys. 119 (17), 9030 (2003).

A. Horikoshi and K. Kinugawa, J. Chem. Phys. 122 (17), 174104 (2005).

R. K. Bryan, Eur. Biophys. J. 18 (3), 165 (1990).

E. Galliccio and B. J. Berne, J. Chem. Phys. 101, 9909 (1994).

E. Galliccio and B. J. Berne, J. Chem. Phys. 105, 7064 (1996).

D. Kim, J. D. Doll, and J. E. Gubernatis, J. Chem. Phys. 106 (4), 1641 (1997).

G. Krilov and B. J. Berne, J. Chem. Phys. 111 (20), 9147 (1999).

E. Rabani, D. R. Reichman, G. Krilov, and B. J. Berne, Proc. Nat. Acad Sci. USA 99, 1129 (2002).

D. R. Reichman and E. Rabani, Phys. Rev. Lett. 87, Art. No. 265702 (2001).

E. Rabani and D. R. Reichman, Europhysics Letters 60, 656 (2002).

E. Rabani and D. R. Reichman, J. Chem. Phys. 116, 6271 (2002). 
D. R. Reichman and E. Rabani, J. Chem. Phys. 116, 6279 (2002).

E. Rabani and D. R. Reichman, Phys. Rev. E 65, Art. No. 036111 (2002).

A. Rahman, K. S. Singwi, and A. Sjolander, Phys. Rev. 126, 986 (1962).

G. L. Squires, Introduction to the Theory of Thermal Neutron Scattering. (Dover Publications, Mineola, NY, 1996).

D. A. McQuarrie, Statistical Mechanics. (University Science Books, Sausalito, CA, 2000).

B. S. Hudson, Vib. Spectrosc 42 (1), 25 (2006).

L. Van Hove, Phys. Rev. 95, 249 (1954).

L. D. Landau and E. M. Lifshitz, Statistical Physics. (Butterworth-Heinemann, Oxford, 1997). 

(2003).

P. A. Frantsuzov and V. A. Mandelshtam, J. Chem. Phys. 121 (19), 9247 (2004).

J. S. Shao and E. Pollak, J. Chem. Phys. 125 (13) (2006). also gives the exact result for the $2^{\text {nd }}$ moment of the incoherent relaxation spectrum, i.e., $\underline{\mu}_{2}(\kappa)$ in Table 1. works slightly better than that of the "vv-std". It has also been noticed that the diffusion constant given by the Kubo-transformed velocity correlation function is a little better than that given by the standard velocity correlation function, based on the TGA/LSC-IVR in our 
experience or the LSC-IVR using the Feynman-Kleinert approximation in Ref. 59. This is partly due to the fact that the TGA/LSC-IVR theoretically gives the exact result of the Kubo-transformed velocity correlation function at $\mathrm{t}=0$ (See Ref. 26) but only an approximation to that of the standard one at $t=0$. We note, however, if the Wigner transform of the operator $\hat{A}^{\beta}$, Eq. (2.12), were carried out exactly, quantities calculated by the Kubo-transformed velocity correlation function would be the same as those calculated by the standard one.

Verkerk, J. Dawidowski, and R. Fernandez-Perea, Phys. Rev. Lett. 84 (23), 5359 (2000).

Chahid, G. Senger, and M. L. Ristig, Phys. Rev. B 47 (22), 15097 (1993). 
(1997).

F. J. Bermejo, B. Fak, S. M. Bennington, R. Fernandez-Perea, C. Cabrillo, J. Dawidowski, M.

T. Fernandez-Diaz, and P. Verkerk, Phys. Rev. B 60 (22), 15154 (1999).

K. H. Andersen, W. G. Stirling, R. Scherm, A. Stunault, B. Fak, H. Godfrin, and A. J. Dianoux, J. Phys.: Condens. Matter 6 (4), 821 (1994).

D. E. Manolopoulos, (Private Communication).

Press, Oxford, 1984). Sacchetti, J. Tomkinson, and M. Zoppi, Appl. Phys. A 74, S64 (2002). http://www.isis.rl.ac.uk/molecularspectroscopy/tosca/. 


\section{Tables}

Table. 1 Two sets of moments given by the three methods based on the LSC-IVR using the TGA as discussed in Section III.

Those that can be analytically exact are marked with " $\checkmark$ " .

\begin{tabular}{lccc}
\hline \hline Moments & Inelastic-std & vv-kubo & vv-std \\
\hline$\mu_{0}(\kappa)$ & $\checkmark$ & $\checkmark$ & $\checkmark$ \\
$\mu_{1}(\kappa)$ & $\checkmark$ & $\checkmark$ & $\checkmark$ \\
$\mu_{2}(\kappa)$ & $\mathbf{x}$ & $\mathbf{x}$ & $\mathbf{x}$ \\
$\underline{\mu}_{0}(\kappa)$ & $\mathbf{x}$ & $\mathbf{x}$ & $\mathbf{x}$ \\
$\underline{\mu}_{1}(\kappa)$ & $\checkmark$ & $\checkmark$ & $\checkmark$ \\
$\underline{\mu}_{2}(\kappa)$ & $\mathbf{x}$ & $\mathbf{x}$ & $\mathbf{x}$ \\
\hline \hline
\end{tabular}




\section{Figure Captions}

Fig. 1 (Color online). Self-parts of the intermediate scattering functions $F_{s}(\kappa, t)=\frac{1}{N} \sum_{i=1}^{N}\left\langle e^{-i \kappa \cdot \mathbf{r}_{i}} \mathrm{e}^{i \boldsymbol{k} \cdot \mathbf{r}_{i}(t)}\right\rangle$ for liquid para-hydrogen at the state point $T=14 \mathrm{~K} ; v=25.6 \mathrm{~cm}^{3} \mathrm{~mol}^{-1}$. Dashed line: $\kappa=0.378 \AA^{-1}$. Dotted line: $\kappa=1.512 \AA^{-1}$. Dot-dashed line: $\kappa=2.646 \AA^{-1}$. Solid line: $\kappa=4.536 \AA^{-1}$.

Fig. 2 (Color online). Incoherent dynamic structure factors for liquid para-hydrogen at the state point $T=14 \mathrm{~K} ; v=25.6 \mathrm{~cm}^{3} \mathrm{~mol}^{-1}$. Solid line: from the Kubo-transformed velocity correlation function (vv-kubo).

Dot-dashed line: from the standard velocity correlation function (vv-std). Dashed line: from the self-part of the intermediate scattering function (inelastic-std).

Fig. 3 (Color online). The first three moments $\mu_{n}(\kappa)$ of the incoherent dynamic structure factors $S_{\text {inc }}(\kappa, \omega)$ shown in Fig. 2. Solid line: exact result. Dashed line with solid circles: from the self-part of intermediate scattering function $F_{s}(\kappa, t)$ (inelastic-std). Hollow circles: from the Kubo-transformed velocity correlation function (vv-kubo). Crosses: from the standard velocity correlation function (vv-std). Dot-dashed line: from the Kubo-transform of $F_{s}(\kappa, t)$ (the self relaxation function $\tilde{F}_{s}(\kappa, t)$ ) by the RPMD method (RPMD-kubo). Hollow squares: from the Kubo-transformed velocity correlation function by the RPMD method (RPMD-vv-kubo).

Fig. 4 (Color online). The relative error of the moment $\mu_{2}(\kappa)$ the incoherent dynamic structure factors $S_{i n c}(\kappa, \omega)$ shown in Fig. 2. Solid line with solid triangles: from the self-part of intermediate scattering function $F_{s}(\kappa, t)$ (inelastic-std). Dashed line with solid circles: from the Kubo-transform of $F_{s}(\kappa, t)$ (the self relaxation function $\tilde{F}_{s}(\kappa, t)$ ) by the RPMD method (RPMD-kubo). 
Fig. 5 (Color online). The first two even moments $\underline{\mu}_{n}(\kappa)$ of the incoherent relaxation function $\tilde{S}_{\text {inc }}(\kappa, \omega)$ based on the incoherent dynamic structure factors $S_{i n c}(\kappa, \omega)$ shown in Fig. 2. Solid line: exact result. Dashed line with solid circles: from the self-part of intermediate scattering function $F_{s}(\kappa, t)$ (inelastic-std). Hollow circles: from the Kubo-transformed velocity correlation function (vv-kubo). Crosses: from the standard velocity correlation function (vv-std). Dot-dashed line: from the Kubo-transform of $F_{s}(\kappa, t)$ (the self relaxation function $\tilde{F}_{s}(\kappa, t)$ ) by the RPMD method (RPMD-kubo). Hollow squares: from the Kubo-transformed velocity correlation function by the RPMD method (RPMD-vv-kubo).

Fig. 6 (Color online). Wave-vector transfer $\kappa(\omega)$ accessible by the TOSCA-II experiment in backward scattering (dashed line) and forward scattering (solid line) as a function of the energy transfer parameter $\omega$ based on the conservation laws, Eqs. (2.3) and (D.1).

Fig. 7 (Color online). Comparison of the LSC-IVR simulations with the inelastic neutron scattering experiment results. along two different kinematic lines in the $(\kappa, \omega)$ plane: (a) forward scattering $\kappa=\kappa_{F}(\omega)$ and (b)backward scattering $\kappa=\kappa_{B}(\omega)$. Solid line: experiment results. Solid squares: from the self-part of intermediate scattering function $F_{s}(\kappa, t)$ (inelastic-std). Hollow circles: from the Kubo-transformed velocity correlation function (vv-kubo). Crosses: from the standard velocity correlation function (vv-std). 


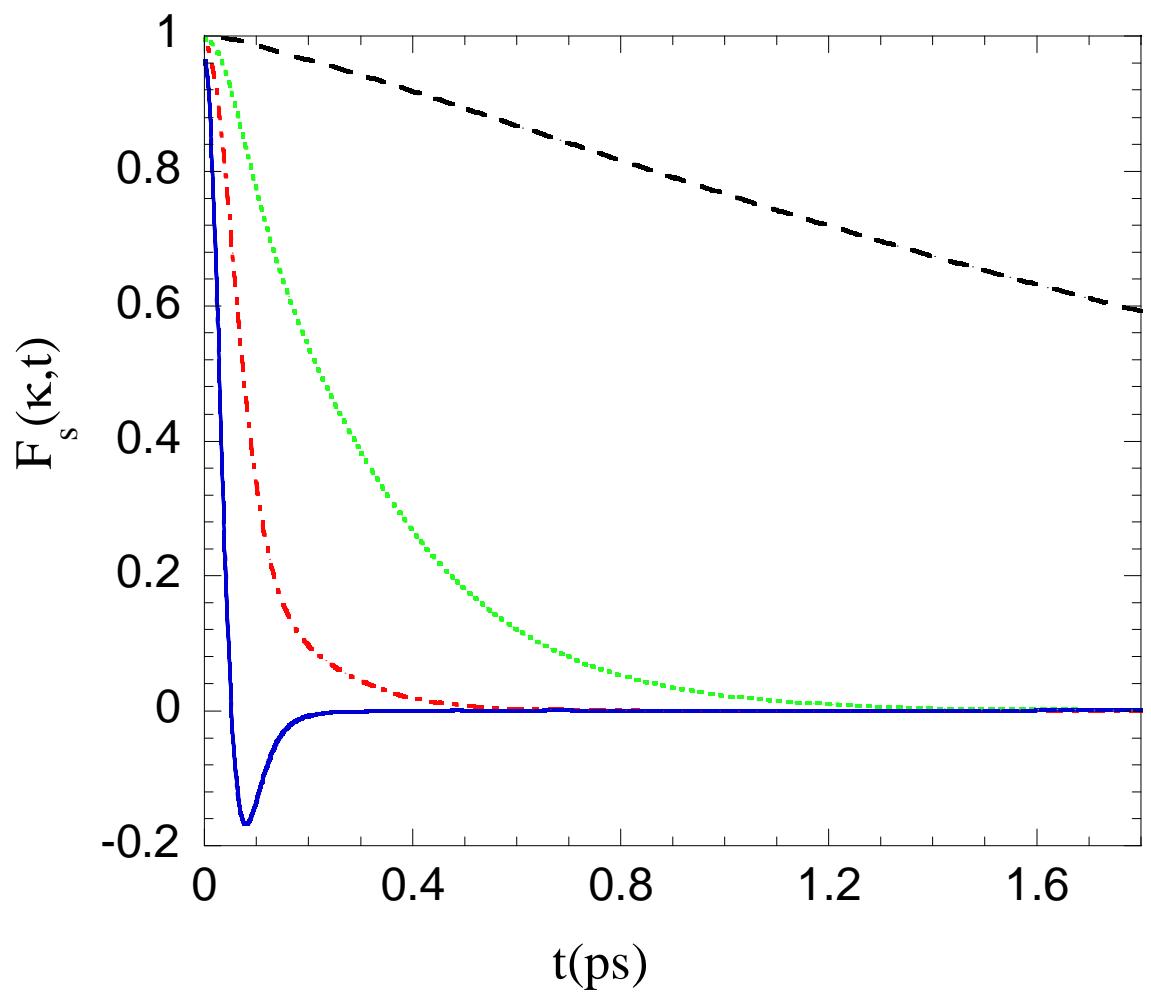

Fig. 1 


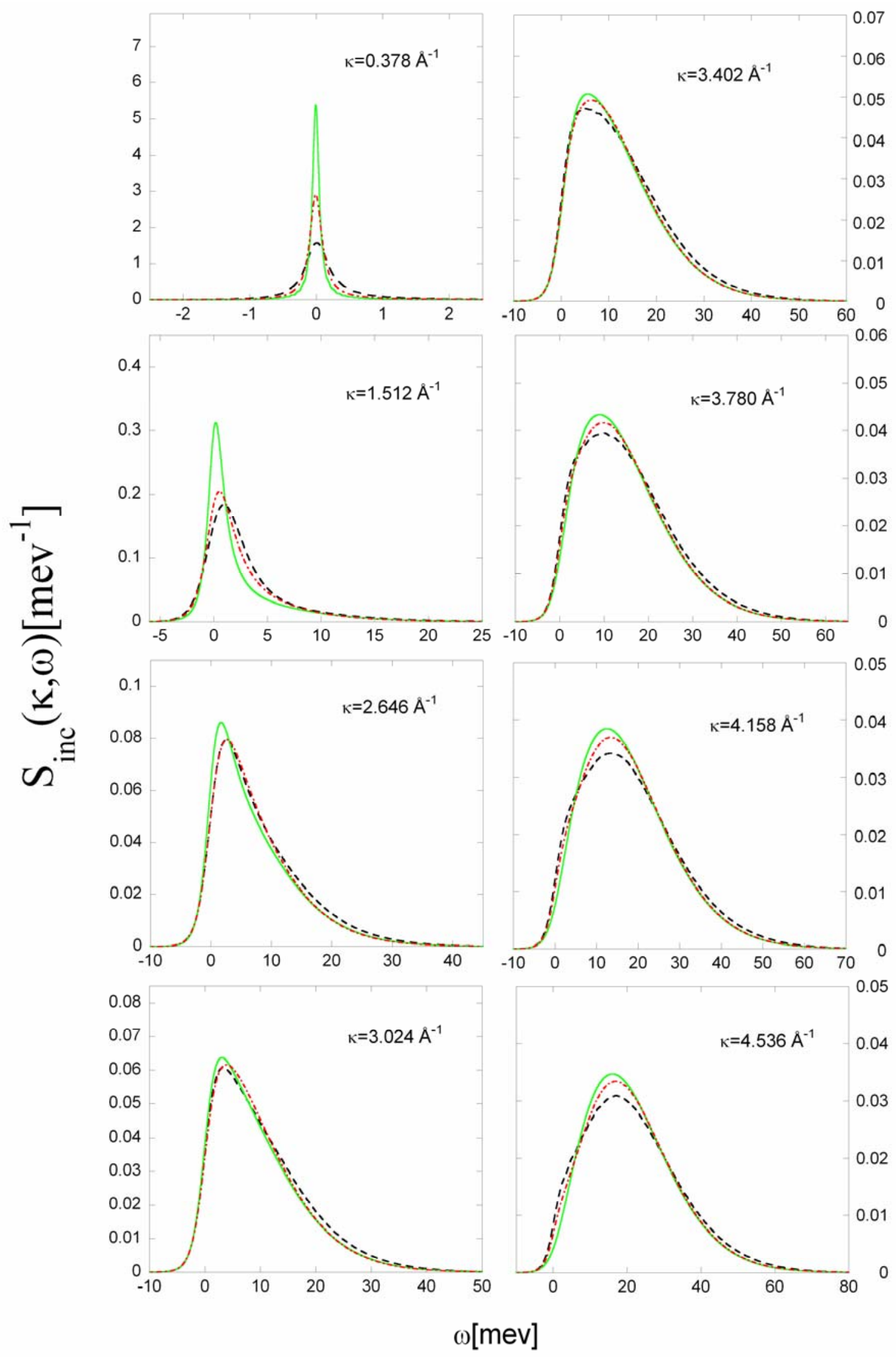

Fig. 2 

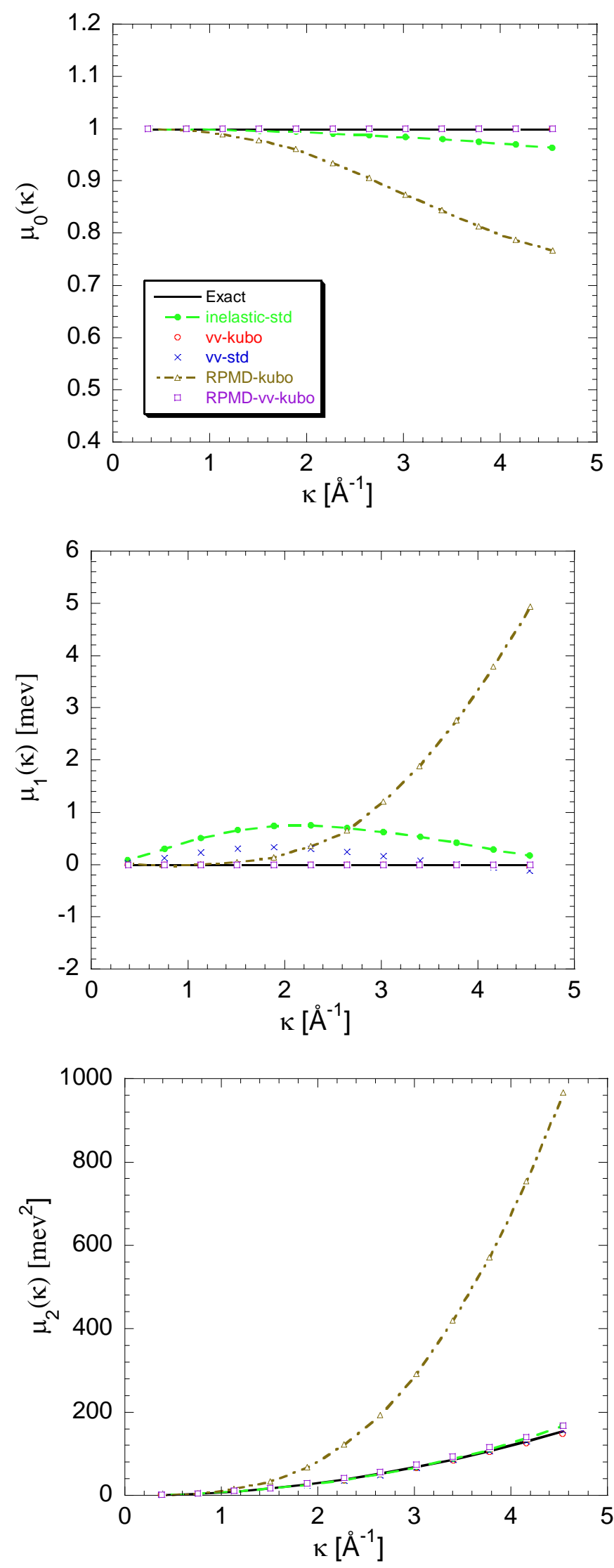

Fig. 3 


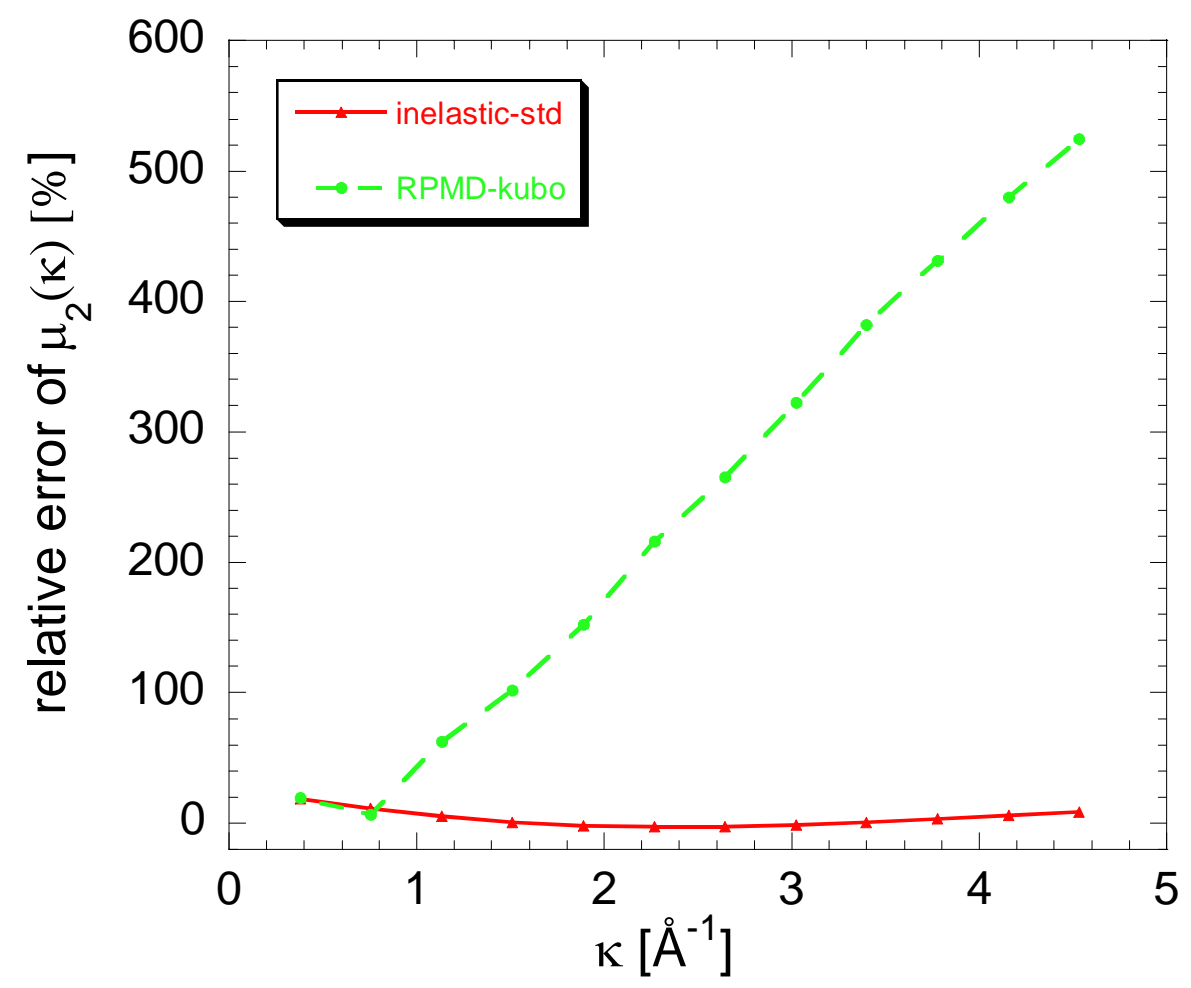

Fig. 4 

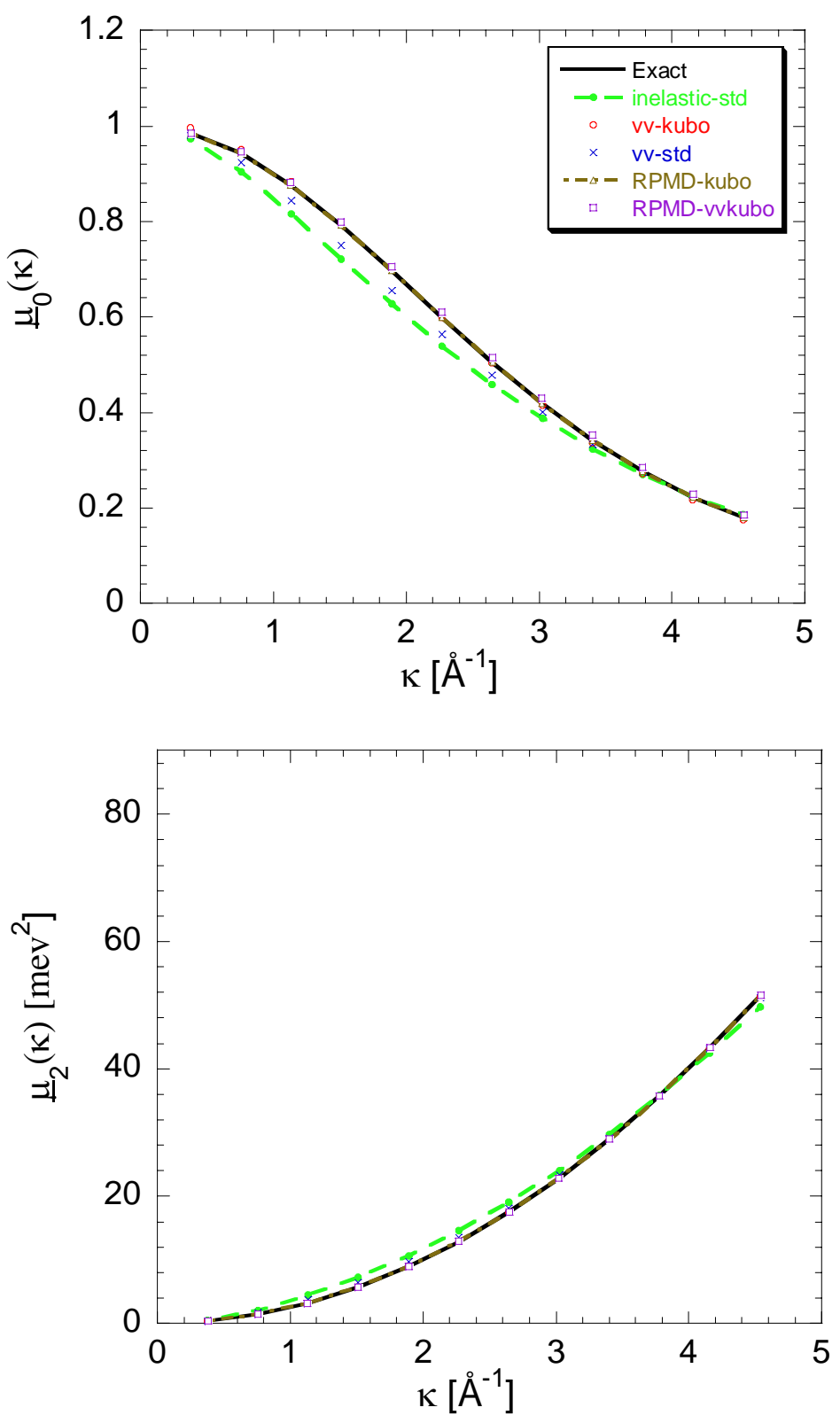

Fig. 5 


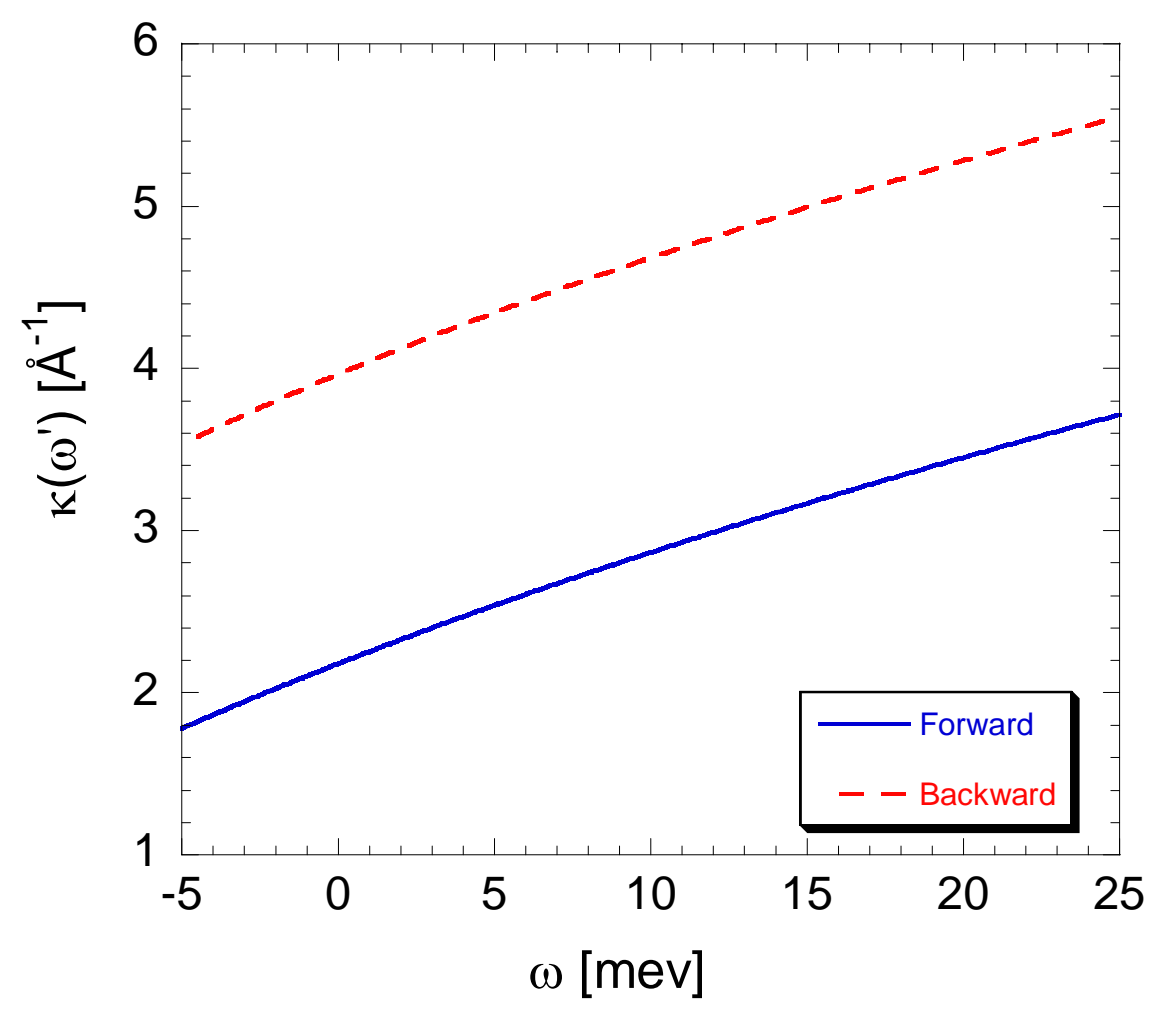

Fig. 6 

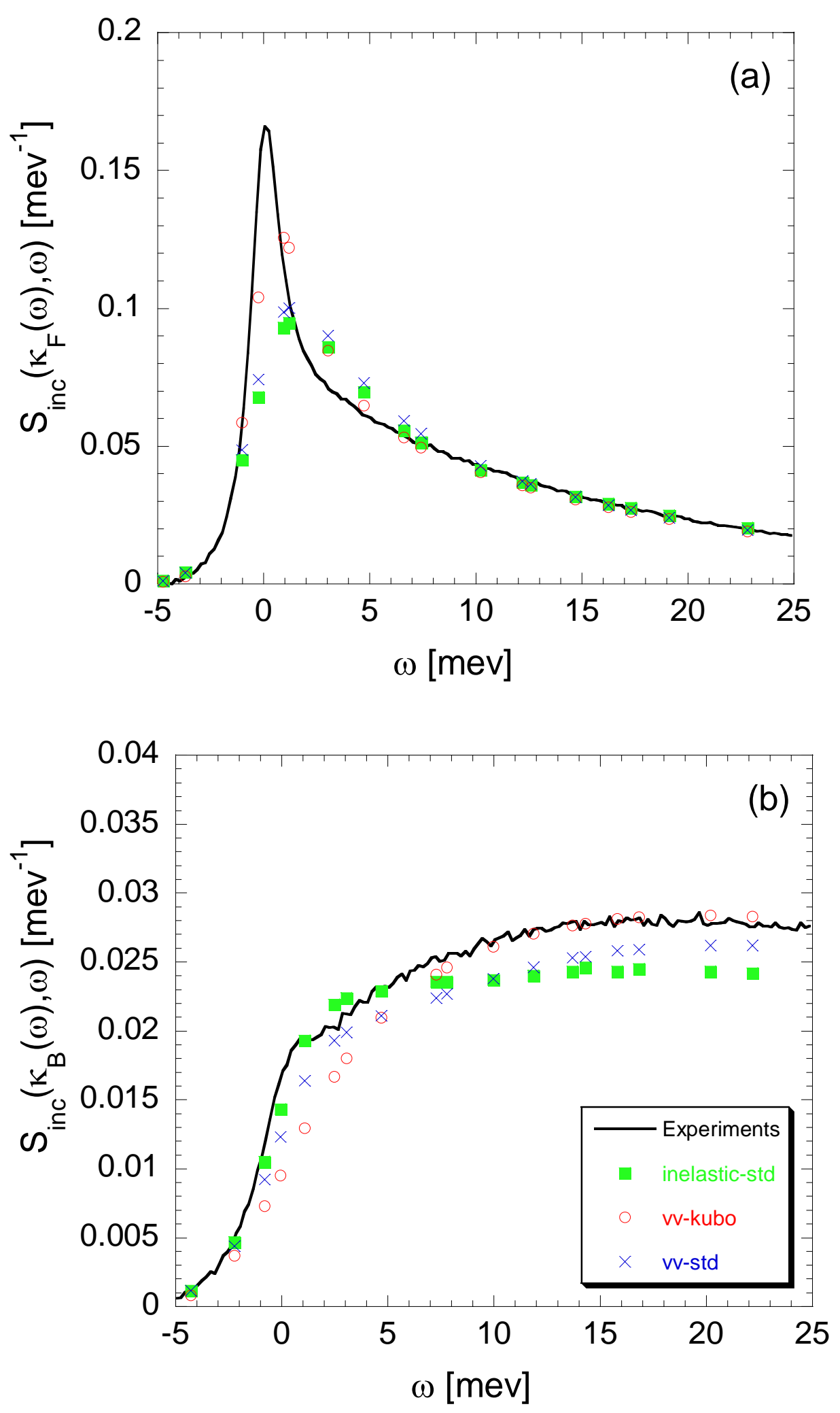

Fig. 7 
